The Impact of Health Changes on Labor Supply: Evidence from Merged Data on Individual Objective Medical Diagnosis Codes and Early Retirement Behavior

Bent J esper Christensen and Malene Kallestrup Lamb 


\title{
The Impact of Health Changes on Labor Supply: Evidence from Merged Data on Individual Objective Medical Diagnosis Codes and Early Retirement Behavior*
}

\author{
Bent Jesper Christensen ${ }^{\dagger}$ \\ Aarhus University and CREATES
}

\author{
Malene Kallestrup Lamb \\ Aarhus University
}

September 2010

\begin{abstract}
People quit the labor force for many different reasons, voluntarily or not, through various arrangements such as unemployment benefits, disability benefits or specially designed early retirement schemes. This paper complements the existing literature by considering a large, register-based sample including objective medical diagnosis codes. We estimate detailed hazard models of duration until retirement, controlling for unobserved heterogeneity and nonparametric baseline hazards, as well as observed heterogeneity through time-varying explanatory variables. These include diagnosis codes, along with a host of demographic, labor market and financial regressors. The panel structure of the data allows following individuals year by year from the age of 50 and precisely measure changes in objectively measured health and other regressors, as well as labor market status. We consider 12 broad, mutually exclusive and exhaustive categories of health diagnoses defined by aggregation across ICD codes. The use of objective medical diagnosis codes should eliminate the justification bias due to self-reports of health, and the large sample size obtained by using register rather than survey data should mitigate the effect of any remaining mismeasurement of true work incapacity. Together, these improvements should help distinguish empirically important effects of health and economic variables on retirement. We distinguish a number of alternative exit routes, in particular, disability, early retirement, unemployment, and others (including out of the labor force and welfare). We estimate both single risk models, lumping all retirement states, and competing risk specifications, including
\end{abstract}

\footnotetext{
*We are grateful to Norma Coe (discussant), Dhaval Dave, Srikanth Kadiyala, and other participants in the 7th iHEA World Congress, Beijing, 2009 , to Susan Averett (discussant) and other participants in the 3rd Biennial ASHE Conference at Cornell University, 2010, and to Diana Weinhold and other seminar participants at London School of Economics for useful comments and suggestions, to Dr. Kristina Bacher Svendsen for help with the medical diagnosis codes, and to the Danish Social Science Research Council and CREATES (funded by the Danish National Research Foundation) for research support. Some of this research was carried out when Christensen was visiting the Economics Department at Harvard University and Kallestrup Lamb was visiting London School of Economics, 
all separate exit routes. Throughout, females are included in the estimations, and we present separate results by gender. We find sizeable differences in retirement behavior across marital status, gender, labor market attachment, occupation, income, and in particular health. We find that the disability retirement exit route that requires specific medical criteria to be met is different from the early retirement route. The latter shares similarities with private pension schemes in a number of countries, including the U.S., where benefits are tied to previous wages, and employers also contribute to this retirement scheme. These differences are pronounced within labor market attachment, income, and in particular health. Furthermore, unemployment followed by early retirement is different from unemployment followed by other programs regarding marital status, gender, income, and health. These comparisons hinge on the competing risk framework. Finally, even when using objective medical diagnosis measures we still find significant effects from health on retirement. Thus, not all health impact on retirement reported in earlier literature was due to justification bias.

JEL Classification: I18, J26, C41.

Keywords: Competing risks, Duration analysis, Grouped data, Justification bias, Objective medical diagnosis codes, Retirement, Unobserved heterogeneity. 


\section{Introduction}

Changes in health may impact individual labor supply decisions, and this has important public policy ramifications. Thus, estimation of the relation between health changes and labor supply is an important empirical issue. A particularly pressing problem is the labor supply of older cohorts approaching the retirement decision and at the same time facing health change hazards that increase with age. As the population ages and the dependency ratio deteriorates, policy makers must look to retain older workers longer in the labor market. Thus, if it is possible to empirically identify specific health related impediments to continued work, then this points to potentially fruitful avenues of investment in improved health.

In this paper, we consider merged register data on early retirement behavior and individual objective medical diagnosis codes for a large, representative Danish sample of older workers drawn at random from the full population. The panel structure of the data allows following individuals year by year from the age of 50 and precisely measure changes in objectively measured individual health, labor market status, and other relevant financial and socio-economic explanatory variables. We consider 12 broad, mutually exclusive and exhaustive categories of health diagnoses defined by aggregation across ICD codes ${ }^{1}$. We estimate detailed hazard models of the impact on duration until retirement of individual health changes and other observed heterogeneity, controlled for as time-varying regressors, and allowing for either parametric or nonparametric specifications of unobserved heterogeneity and baseline hazards, as well as potentially heterogeneous labor market exit routes.

Unfortunately, estimates of the effect of health on retirement behavior are plagued by justification and attenuation biases stemming from endogenous self-reporting of health conditions in surveys and classical measurement error, respectively. Although the two biases work in opposite directions, there is no reason to expect that they should cancel exactly in any given data set. Furthermore, the combined (i.e., net) bias is likely to be non-neglible in the existing literature, as this is largely restricted to surveys, implying that health status is necessarily self-reported, and sample size is modest. The size of the bias in self-reported health measures is documented in Benitez-Silva et al. (2004) and Baker et al. (2004). In surveys where subjects are asked both about health and labor market status, a justification or rationalization bias is likely to arise, as individuals tend to use poor health as an excuse for early retirement. This implies an upward bias in measurement of the severity of the health condition. For purposes of estimating the impact of health changes on retirement behavior, the justification bias leads to an endogeneity problem, as well, since retirement (the endogenous variable) drives the self-report on health. The justification bias in retirement studies has been discussed by Anderson et al. (1985), Bound (1991), and Dwyer et al. (1999). The policy issue is that if the estimated effect of health changes on the propensity to withdraw from the labor market is exaggerated, then so is the apparant impact on labor force size of policy decisions regarding investment in the health industry. A recent study suggesting that failing health induces workers to retire is McGarry (2004).

Without access to register data including medical diagnosis codes, some researchers have considered more

\footnotetext{
${ }^{1}$ We use the International Statistical Classification of Diseases and Related Health Problems, 10th Revision (ICD-10), a coding of diseases and signs, symptoms, abnormal findings, complaints, social circumstances and external causes of injury or diseases, as classified by the World Health Organization (WHO). The code set allows more than 22,000 different codes.
} 
objectively measured health indicators, e.g., self-reports of specific diseases, rather than self-reports of overall work incapacity. These studies tend to find smaller effects on retirement, consistent with the possibility that the justification bias has been reduced. Nevertheless, Baker et al. (2004) directly compares such specific self-reports to medical records and finds that significant biases remain. Furthermore, as all measures are liable to suffer from additional classical (random) error leading to attenuation bias in the opposite (downward) direction in retirement models, reduction in the justification bias may make the opposing biases even less likely to cancel (Bound (1991)). In a recent study, Datta Gupta et al. (2010) consider a combined survey-register match-up including both self-reported health and medical diagnosis codes, along with planned (future) retirement dates and additional explanatory variables. They find biases in planned retirement impacts based on self-reports, even of more objective types, in particular in case of illnesses with more diffuse symptoms. Since planned as opposed to actual retirement behavior is studied, and health status is only reported at one point in time, the analysis does not reveal how subsequent changes in health impact actual retirement behavior over time. Thus, it is important to consider a large, register-based data set including physician diagnoses and actual retirement dates in order to simultaneously reduce both biases.

In the present paper, we consider actual rather than planned retirement dates, and use period by period changes in objective health measures based on medical diagnosis codes and other explanatory variables as timevarying regressors. This allows estimation of the labor supply response at the time of the onset of the health problem in question. As we use merged population registers as opposed to a survey match-up, we are able to follow a larger sample of individuals (9,329 individuals, for 78,150 person-year observations in the estimations) and identify the actual timing of health, financial, demographic, and labor market status events. The large longitudinal panel should provide for estimates of the determinants of retirement that are precise relative to those obtained using different types of survey instruments (including general or more specific self-reports), with the necessary limitation to smaller samples and largely cross-sectional differences in health levels, and afflicted with both justification and (greater) attenuation bias. Indeed, to the extent that our use of objective diagnosis codes eliminates the justification bias and reduces the error in measurement of true work incapacity, leaving only a reduced (through larger sample size) attenuation bias in the estimated impact of health changes on labor supply, our estimates should, if anything, be on the conservative side.

We distinguish a number of alternative exit routes, in particular, disability, early retirement, unemployment, and others (including out of the labor force and welfare). Details on the available retirement programs during the sample period are in the appendix. We estimate both single risk models, lumping all retirement states, and competing risk specifications, including all separate exit routes. In spite of the different nature of the alternative exit routes, the competing risk approach has rarely been applied in retirement research. Butler, Anderson, and Burkhauser (1989) model reentry into work from retirement versus death using a competing risk approach applied to survey data on men and unmarried women (RHS). Our empirical results confirm that there are important differences between exit routes in the retirement case.

Throughout, both males and females are included in the estimations, and we present separate results by gender in case of the single risk models, while including gender dummies in the less parsimonous specifications. 
We do not consider old age pension, avaliable at age 67 in our sample period, but focus on alternative earlier retirement options and follow individuals from age 50 to 66 . Disability retirement requires specific medical criteria to be met, as assessed by a doctor. It is natural to expect some of the conditions associated with the diagnosis codes to increase the rate of disability retirement. In addition, we control for receipt of sickness benefits, as an additional health indicator. Early retirement in our sample corresponds to post employment wages, a labor market pension scheme available from age 60 conditional on preceding regular contributions for 10 to 25 years (depending on year of retirement) to an unemployment insurance fund. Thus, early retirement here shares similarities with private pension schemes in a number of countries, including the U.S.. Benefits are tied to previous wages, and employers also contribute to this retirement scheme, which is financially attractive, but unavailable once the disability route has been selected. In our estimations, we also control for UI-fund membership, and, through the nonparametric baseline hazard, for age.

For the age groups considered, onset of unemployment typically represents exit from the labor market, too. In many cases, a spell of unemployment is followed by disability or early retirement. Unemployment insurance benefits are available only in case of preceding UI-fund membership, too, and exceed welfare (social assistance) by about one third. In our estimations, the unemployment state represents receipt of UI benefits. Welfare recepients are included in the destination 'Other'. UI benefits may generally be received only for a maximum of four years, and are then replaced by welfare, but in Denmark in our sample period, individuals aged 5559 were allowed to continue on UI benefits beyond the four-year period, thus allowing a smooth transfer to early retirement upon reaching the eligibility age of 60 . This apparantly exceedingly generous scheme might be related to high unemployment rates during the period, and a government goal of reducing the rates among the young. In our context, this generates an interest in the combined exit route of unemployment followed by early retirement, implicitly encouraged by the system. If health, demographic, and financial determinants of retirement have different impacts on the hazard for this combined exit route and the hazard for the route via unemployment not followed by early retirement, then it indicates that the combined route is voluntary, thus reinforcing the interpretation of this form of unemployment as a part of retirement planning.

The rest of this paper is laid out as follows. The data are introduced in Section 2. Section 3 presents the econometric methodology, and Section 4 discusses the empirical results. Section 5 concludes.

\section{Data}

The full data base contains annual observations on all individuals in Denmark above 18 years of age for the period 1985-2001, with measurement in November each year. The data are based on administrative registers and contain no survey element. We have information on various individual demographic and socio-economic characteristics, health, and labor market status. This enables us to identify individual transitions between different labor market states and health events on an annual basis.

The sample is drawn at random from the set of all individuals who are exactly 50 years old and active in the labor market in 1985. We follow them until retirement, death, or end of study period, whichever is first. By excluding the age group 18-49 we avoid the part of early retirement that is associated with a limited job 
carreer and loose labor market attachment. We focus on individuals that actually retire from the labor market after an active career. We exclude those who are outside the labor market the first time we observe them. We include the employed, and all unemployed workers who are actively looking for a job (namely, receive benefits and therefore satisfy certain job search requirements).

The duration variable takes the value one in the base year and we then follow a person until he or she reaches the age of 66 and is no longer at risk of retiring early. This implies a maximum duration of 17 years. A single spell describes the number of years the individual actively participates in the labor market after the age of 50 . For example, a person could be employed, unemployed, reemployed, then retire, and the total spell will be the sum of years spent in the three first states. Thus, the duration is defined as

$$
\text { Duration }=\text { Age }-49
$$

Right censoring occurs at age 67 or in the event of death. This feature is incorporated in the likelihood function. By definition, spells start at age 50, so there is no left censoring. Starting at age 50 and conditioning on labor market participation at this age implies that our results are not representative for the full population, including those who tend to enter non-participation and retirement states even before 50. The latter group is less interesting from the point of view of policy reforms intended to keep older workers longer on the labor market. Given our focus on the group participating at 50, there is no need to consider an alternative flow sampling procedure, since adding participating individuals who turn 50 in the subsequent years does not change the representativeness relative to the full population. The final number individuals in the sample is 9,329 .

Table 1 shows the duration data. Two different measures are presented. The first is the total number of observed spells for all persons in the sample. The second is the number of observed exits to an early retirement program. The difference between the two represents exits due to death. The distribution of exit is well spread out across different durations. The three smallest cell counts are for the two shortest and the longest duration. For numerical purposes, the first two durations are combined when we conduct the semi-parametric analysis below, and the last duration length is excluded (right censoring occurs a year early), since individuals who participate after age 66 in all but 6 cases then continue until 67, the official old age pension (OAP) age during the sample period.

We are interested in the transition to the different retirement schemes, and especially how this depends on the personal characteristics in the years before retirement. Since we only observe that an exit to retirement has occurred sometime within a given year, we use explanatory variables for time $t-1$ (previous November) to explain the retirement hazard at time $t$. This is in order to avoid a potential endogeneity issue arising if the value of a given characteristic is influenced by the retirement event.

Table 2 contains descriptive statistics of the explanatory variables. We report the means and standard deviations over all person-years. All variables are allowed to vary during the labor market spell, and may be used as time-varying regressors, and all explanatory variables are normalized to the $[0,1]$ interval for estimation purposes. Panel A reports statistics for standard labor market and socio-economic background variables typically used in retirement studies. Panel B reports statistics for financial and health variables, including the medical 
diagnosis codes unique to this study.

The geographical variable city is an indicator variable taking the value one for residence within the Copenhagen metropolitan area. The variable married takes the value one if the individual has an identified partner. Being married therefore includes persons that are married or cohabitating. Male is a dummy variable for gender. In our sample, $80 \%$ are married or cohabiting. The are more males (57\%) than females in the sample, due to conditioning on participation.

The extent of the labor market attachment is important due to the different rules for the full-time and part-time employed. We construct a dummy variable taking the value one if the individual is working full-time and zero otherwise. Two thirds of the sample consists of full-time workers. The yearly unemployment rate is based on the number of hours the individual has been unemployed relative to the number of possible hours worked. On a yearly basis it can indicate one long unemployment spell or several periods of unemployment. The rate is $6 \%$ on average, but varies considerably across the sample, with a standard deviation of $19 \%$. The variable experience defines the individual's work experience since 1980. A full-time employee can achieve a maximum of 1 and a part-time employee .75. The indicator variable sickness pay is constructed on the basis of received sickness benefit and takes the value one if the individual has received this type of benefit during the year. ${ }^{2}$ Being a member of an UI-fund is relevant in relation to the eligibility criteria for some of the early retirement schemes and takes the value one if such a membership exists.

Education is divided into five categorizes: Basic, Vocational, Short, Medium, and Long, and is defined on basis on the individual's highest completed education level. Basic refers to primary or high school, only. Short, Medium, and Long are all higher educations beyond the high school level. Long includes all university degrees at the Bachelor level or higher, as well as engineers and architects with five years or longer programs. Short and Medium refer to non-university degrees, with Short including less academic programs than Medium, and the latter typically requiring about 4 years after high school. Examples of educations under Short include real estate broker, actor, correspondent, technician with some training beyond vocational, laboratory worker, etc. Medium includes school teacher, journalist, librarian, accountant, nurse, midwife, social worker, some engineering, etc. Since we look at older cohorts, only $4 \%$ have long higher education in our sample, and nearly half the sample has only basic education, while about one third has vocational training.

Job characteristics are described through occupational indicators: Self-employed, Assisting spouse, Salaried worker at high, medium, and low level, and Unskilled. These are broad categories, with 10\% or more in each, except only $3 \%$ in Assisting spouse. Among Salaried workers, the high level includes directors, managers, etc., medium level is other office personnel, and low level is skilled blue collar workers.

There are three financial indicators, all deflated to 2000 levels and measured in logarithms, ${ }^{3}$ namely own income, ${ }^{4}$ total household income, and household net wealth based on calculations from the IRS. As expected,

\footnotetext{
${ }^{2}$ The first two days of illness are covered by the employer, and less than $1 \%$ of the sample actually experience longer sickness spells and receive sickness pay, but the variable is nevertheless strongly significant in several of our specifications below.

${ }^{3}$ In case of zero or negative values, the logarithm is set equal to zero. Various alternative truncation and transformation schemes made little difference to the results.

${ }^{4}$ As an alternative, the hourly wage has also been considered. Due to a relatively large number of missing wage observations, own income is preferred.
} 
the standard deviation is greater on wealth than on the income variables. Since we control for household income and wealth, as well as marital status, we do not separately control for spouse's retirement status.

In addition to these standard background characteristics, we have information on the individual's health situation over time through diagnosis codes, and this is the unique feature of the present study. The health data are drawn from the Danish National Registry for Patients and includes information about admissions, actual diagnoses, treatments, and discharges made for all patients who have been in contact with clinical hospital departments in Denmark during the period. After 1994, information about out-patient treatments or emergency room visits is also available. The essential feature is that we have information about the truly objective medical diagnoses made at the time of a hospital discharge, and thereby avoid the justification bias related to self-reported health measures.

Within each year we have multiple observations for a given patient since the possibility of several admissions exists (approximately one third of the patients experience more than one admission within a given year). Furthermore, in relation to an admission, the patient is diagnosed with a main condition and possibly several additional conditions. The different diagnoses are organized in relation to WHO's international classification of diseases (ICD). From 1980 through 1993, ICD-8 was used, and from 1994 through 2001 ICD-10. All this information is summarized in 12 dummy variables, each taking the value one if a person has been diagnosed with a disease in the associated category within the year. Thus, multiple dummy variables can take the value one simultaneously. The categories are: (1) Malignant cancer (includes leukemia, melanoma, and other malignant cancers); (2) Benign cancer (various types of tumors); (3) Endocrine, nutritional, and metabolic diseases (e.g., diabetes, obesity, etc.); (4) Mental and behavioral disorders (dementia, delirium, skizophrenia, stress-related disorders, etc.); (5) Diseases of the nervous system and sense organs (Alzheimer's, Parkinson's, epilepsy, sclerosis, migraine, apnoea, cataract, hearing loss, etc.); (6) Diseases of the circulatory system (ischaemic and other heart diseases, angina pectoris, acute rheumatic fever, high blood pressure, hypertension, stroke and other cerebrovascular diseases); (7) Diseases of the respiratory system (influenza, pneumonia, acute and chronic bronchitis, asthma, and other lung diseases); (8) Diseases of the digestive system (gastric ulcer, hernia, diseases of the liver and gallbladder, etc.); (9) Diseases of the genitourinary system (kidney stone, renal failure, other diseases of the urinary system and genital organs); (10) Diseases of the musculoskeletal system and connective tissue (arthritis, osteoarthritis, Lyme disease, herniated disc, lumbago, osteoporosis, sclerosis, rheumatism, gout); (11) Injury, poisoning and certain other consequences of external causes (bone fractures, dislocations, etc.); (12) Other diseases. Both main and additional diagnoses are included, since it is just as likely that it is an additional diagnosis that influences the decision to retire early. Furthermore, the number of days of treatment, number of diagnoses, and number of admissions within a given year are included.

Figure 1 shows the nonparametric estimate of the full sample retirement hazard function $h$ and associated confidence bands. Specifically, in the annual data, the possible durations until retirement are $j=1, \ldots, 17$. Define for each $j$ the quantites

- $d_{j}$ : the number of individuals observed to retire in year $j$

- $m_{j}$ : the number of individuals whose observed spell is censored in year $j$ 
- $n_{j}$ : the number of individuals not yet retired (i.e., still at risk) in year $j-1$. This group consist of those who have a completed or censored spell of duration $j$ or longer:

$$
n_{j}=\left(m_{j}+d_{j}\right)+\left(m_{j+1}+d_{j+1}\right)+\ldots . .+\left(m_{17}+d_{17}\right) .
$$

Then the hazard rate is estimated as the number of individuals retiring relative to the number at risk,

$$
\widehat{h_{j}}=\frac{d_{j}}{n_{j}},
$$

i.e., the $j$ 'th increment to the Nelson (1972) and Aalen (1978) estimator $\widehat{H_{j}}=\sum_{i \leq j} d_{i} / n_{i}$ of the cumulative hazard function. The corresponding Kaplan and Meier (1958) product-limit estimate of the survivor function is

$$
\widehat{S_{j}}=\prod_{i \leq j}\left(1-\frac{d_{i}}{n_{i}}\right) .
$$

Johansen (1978) provides an interpretation of this as a maximum likelihood estimator in a wide variety of settings. The asymptotic variance of $\widehat{S_{j}}$ is estimated using the method of Greenwood (1926),

$$
\operatorname{Var}\left(\widehat{S_{j}}\right)={\widehat{S_{j}}}^{2} \sum_{i \leq j} \frac{d_{i}}{n_{i}\left(n_{i}-d_{i}\right)},
$$

and the corresponding variance of the estimated hazard (3) is

$$
\operatorname{Var}\left(\widehat{h_{j}}\right)=\frac{d_{i}\left(n_{i}-d_{i}\right)}{n_{i}^{3}},
$$

which forms the basis of the confidence band in Figure 1.

There is positive duration dependence initially, and a clear peak at age 60 (duration 10), reflecting the early retirement program. There is a second (lower) peak at age 63. The shape of the overall hazard function is in accordance with earlier findings on early retirement in Denmark (Pedersen and Smith (1991), An, Christensen and Datta Gupta (2004)). Of course, the nonparametric estimates only reveal average transition rates out of work, not how these vary with health and other characteristics of the sample.

\section{Grouped Duration Analysis}

Although our data are grouped in that duration is measured in whole years, it is useful for model specification purposes to start the analysis with the underlying continuous time duration $T$. The distribution of $T$ is characterized by the conditional hazard rate out of employment, given time-varying regressors $x_{t}$,

$$
h\left(t \mid x_{t}\right)=\lim _{d t \rightarrow 0} \frac{\operatorname{Pr}\left(t \leq T<t+d t \mid T \geq t, x_{t}\right)}{d t},
$$

assuming the limit exists. Of course, with grouped data, the continuous time hazard function is not nonparametrically identified. The grouped data approach summarizes the information on staying in the initial state or exiting in each time interval in a sequence of binary outcomes, see Prentice and Gloeckler (1978), Kiefer (1988, 1990), Han \& Hausman (1990), Lancaster (1990), and McCall (1994). Thus, divide the time line into $K+1$ 
intervals, $\left[0, t_{1}\right),\left[t_{1}, t_{2}\right), \ldots \ldots .,\left[t_{K-1}, t_{K}\right),\left[t_{K}, \infty\right)$, where the $t_{k}$ are known constants. ${ }^{5}$ Any duration falling into the last interval, $\left[t_{K}, \infty\right)$, is censored at $t_{K}$. Given explanatory variables at level $x_{k}$ over the course of the $k^{\prime}$ th interval, the conditional probability that the duration $T$ is greater than $t_{k}$ given that it is greater than $t_{k-1}$ is

$$
\begin{aligned}
P\left(T>t_{k} \mid T>t_{k-1}, x_{k}\right) & =\exp \left[-\int_{t_{k-1}}^{t_{k}} h\left(t \mid x_{k}\right) d t\right] \\
& =\alpha_{k}\left(x_{k}, \theta\right) .
\end{aligned}
$$

Thus, given $T$ is not in one of the $k-1$ first intervals, the probability that it is in the $k^{\prime}$ th interval is $1-\alpha_{k}$, and with probability $\alpha_{k}$ it is in a later interval, so $\alpha_{k}$ and $1-\alpha_{k}$ are the discrete time survivor and hazard, respectively. The individual contribution to the likelihood function for an individual with duration in the $k^{\prime}$ th interval and observed regressors $x_{j}, j=1, \ldots, k$, is

$$
\begin{aligned}
L(\theta, k, x) & =\left[\left(1-\alpha_{k}\left(x_{k}, \theta\right)\right) \prod_{j=1}^{k-1} \alpha_{j}\left(x_{j}, \theta\right)\right]^{d}\left[\prod_{j=1}^{k} \alpha_{j}\left(x_{j}, \theta\right)\right]^{1-d} \\
& =\left(1-\alpha_{k}\left(x_{k}, \theta\right)\right)^{d} \alpha_{k}^{1-d}\left(x_{k}, \theta\right) \prod_{l=1}^{k-1} \alpha_{l}\left(x_{l}, \theta\right)
\end{aligned}
$$

where $d=1$ if the duration is uncensored and zero otherwise.

The $\log$ likelihood function for a sample of $n$ individuals, with the $i$ 'th retiring in the $k_{i}{ }^{\prime}$ th interval, is therefore

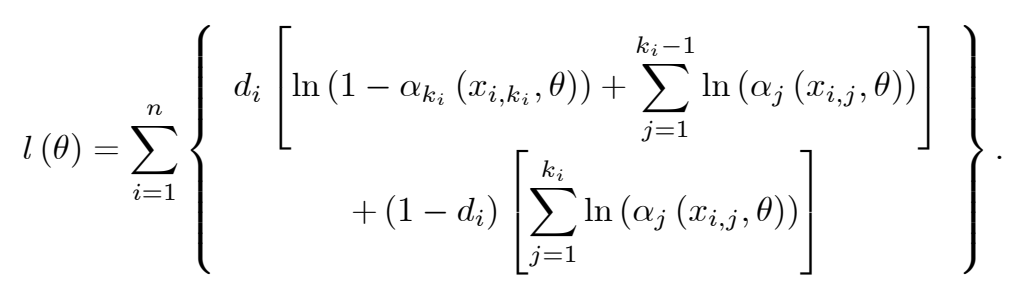

Here, $\theta$ is the unknown parameter vector to be estimated, and $x_{i, j}$ is the relevant vector of explanatory variables for individual $i$ in the $j^{\prime}$ th interval. In each of our specifications, the MLE for $\theta$ is computed by maximizing (10) using the BFGS algorithm (Broyden (1970), Fletcher (1970), Goldfarb (1970), Shanno (1970)), and asymptotic standard errors are based on the diagonal of the inverse of the matrix summing the outer products of the individual score contributions, see Berndt, Hall, Hall, and Hausman (1974). The convergence tolerance for the score with respect to each coefficient is set to 1.0e-4.

Our alternative models differ with respect to the specification of $\alpha_{k}$. In the Cox (1972) proportional hazard case (see also Kalbfleisch and Prentice (1973)), we assume that the hazard is the product of a baseline hazard, $\lambda(t)$, depending only on time, and a function specifying the effect of the explanatory variables. The standard form of the latter is the exponential in $x_{t}^{\prime} \beta$, where $\beta$ are regression coefficients of interest, yielding

$$
h\left(t \mid x_{t}\right)=\lambda(t) \exp \left(x_{t}^{\prime} \beta\right) .
$$

\footnotetext{
${ }^{5}$ We could have $t_{k}=k$, but unequally spaced intervals are allowed.
} 
We still allow for time-varying covariates $x_{t}$, so the hazard may vary over time due to changes in both factors in (11). In this case,

$$
\begin{aligned}
\alpha_{k}\left(x_{i, k}, \theta\right) & =\exp \left[-\int_{t_{k-1}}^{t_{k}} h\left(t \mid x_{i, t}\right) d t\right] \\
& =\exp \left[-\int_{t_{k-1}}^{t_{k}} \lambda(t) \exp \left[x_{i, k}^{\prime} \beta\right] d t\right] \\
& =\exp \left[-\exp \left(x_{i, k}^{\prime} \beta\right) \cdot \Lambda_{k}\right],
\end{aligned}
$$

where $\Lambda_{k}=\int_{t_{k-1}}^{t_{k}} \lambda(t) d t$. This is inserted in (10) to construct the log likelihood function. Note that a parameter $\Lambda_{k}$ is associated with each interval for the grouped data, i.e., $\theta=\left(\beta, \Lambda_{1}, \ldots, \Lambda_{K}\right)$, following Kiefer (1988). The approach is semiparametric, allowing a general unspecified baseline hazard $\lambda_{0}(\cdot)$, and estimating this only up to its integrals $\Lambda_{k}$ over the $K$ intervals given by the data collection mechanism. We allow the regressors $x_{t}$ to vary across the $K$ intervals, although we take it to be constant within each interval, in order to be able to take it outside the integral in (12) above, and since in any case our explanatory variables are only observed at the same frequency as the durations, once per interval (year). The alternative partial likelihood approach of Cox (1975) similarly takes the regressors to be constant within intervals and avoids dealing with the baseline altogether by estimating $\beta$ off the ranks of the durations, but it requires a rule for breaking ties among the durations, using, e.g., the Breslow (1974) or Efron (1977) approximations to the partial log likelihood function, and unlike our approach it does not allow an investigation of the shape of the baseline hazard.

A restricted model specification is obtained by parametrizing the baseline hazard, deriving the resulting form for $\Lambda_{k}$ and hence $\alpha_{k}$, and estimating the parameters of the baseline specification along with $\beta$. The exponential distribution with its associated constant hazard,

$$
\lambda(t)=\gamma,
$$

is a benchmark case in duration analysis since it exhibits no duration dependence. In this case,

$$
\Lambda_{k}=\gamma\left(t_{k}-t_{k-1}\right)
$$

to be inserted in $\alpha_{k}$, so $\theta=(\beta, \gamma)$ in the estimation. A natural generalization allowing for duration dependence is the Weibull specification. If $T^{\alpha}$ is exponential then $T$ is Weibull with shape parameter $\alpha$. This model has been applied frequently, e.g., to the duration of unemployment spells, see Lancaster (1979), Nickell (1979), and Kiefer (1988). The Weibull baseline hazard is given by

$$
\lambda(t)=\alpha t^{\alpha-1} \gamma
$$

with scale parameter $\gamma$ and duration dependence $\alpha-1$, i.e., this can be positive or negative, corresponding to monotonically increasing or decreasing baseline, and the special case $\alpha=1$ is the exponential. In the Weibull case,

$$
\Lambda_{k}=\gamma\left(t_{k}^{\alpha}-t_{k-1}^{\alpha}\right)
$$

again used in $\alpha_{k}$, so $\theta=(\beta, \gamma, \alpha)$. 


\subsection{Unobserved Heterogeneity}

The models so far only allow for observed heterogeneity which is controlled for through the regressors $x_{t}$. We now consider in addition unobserved heterogeneity entering via an individual-specific term $v$, so that the conditional hazard is of the form $h\left(t \mid x_{t}, v\right)$. In the proportional hazard case, (11) is generalized to

$$
h\left(t \mid x_{t}, v\right)=v \cdot \lambda(t) \exp \left(x_{t}^{\prime} \beta\right) .
$$

Consequently, conditionally on the heterogeneity term, the discrete time survivor (12) becomes

$$
\alpha_{k}\left(x_{i, k}, v_{i}, \theta\right)=\exp \left[-v_{i} \cdot \exp \left(x_{i, k}^{\prime} \beta\right) \cdot \Lambda_{k}\right] .
$$

The likelihood function is formed by integrating out the unobserved $v_{i}$, using a parametric or nonparametric distributional assumption. Writing $G\left(v \mid x_{t}, \theta\right)$ for the conditional heterogeneity distribution, the general form of the individual contribution to likelihood is obtained by substituting (18) in (9),

$$
\begin{aligned}
L(\theta, k, x)= & \int\left(1-\exp \left[-v \cdot \exp \left(x_{k}^{\prime} \beta\right) \cdot \Lambda_{k}\right]\right)^{d} \exp \left[-v \cdot \exp \left(x_{k}^{\prime} \beta\right) \cdot \Lambda_{k}\right]^{1-d} \\
& \cdot \prod_{l=1}^{k-1} \exp \left[-v \cdot \exp \left(x_{l}^{\prime} \beta\right) \cdot \Lambda_{l}\right] G(d v \mid x, \theta)
\end{aligned}
$$

and again $d=1$ if the labor market spell is not right censored. Rewriting this expression, we have

$$
\begin{aligned}
L(\theta, k, x)= & \int\left(\prod_{l=1}^{k-1} \exp \left[-v \cdot \exp \left(x_{l}^{\prime} \beta\right) \cdot \Lambda_{l}\right]-\prod_{l=1}^{k} \exp \left[-v \cdot \exp \left(x_{l}^{\prime} \beta\right) \cdot \Lambda_{l}\right]\right)^{d} \\
& \cdot\left(\prod_{l=1}^{k} \exp \left[-v \cdot \exp \left(x_{l}^{\prime} \beta\right) \cdot \Lambda_{l}\right]\right)^{1-d} G(d v \mid x, \theta) \\
= & \int\left(\exp \left\{-v \cdot \sum_{l=1}^{k-1}\left[\exp \left(x_{l}^{\prime} \beta\right) \cdot \Lambda_{l}\right]\right\}-\exp \left\{-v \cdot \sum_{l=1}^{k}\left[\exp \left(x_{l}^{\prime} \beta\right) \cdot \Lambda_{l}\right]\right\}\right)^{d} \\
& \cdot\left(\exp \left\{-v \cdot \sum_{l=1}^{k}\left[\exp \left(x_{l}^{\prime} \beta\right) \cdot \Lambda_{l}\right]\right\}\right)^{1-d} G(d v \mid x, \theta) \\
= & \left(M_{G}\left\{-\sum_{l=1}^{k-1}\left[\exp \left(x_{l}^{\prime} \beta\right) \cdot \Lambda_{l}\right]\right\}-M_{G}\left\{-\sum_{l=1}^{k}\left[\exp \left(x_{l}^{\prime} \beta\right) \cdot \Lambda_{l}\right]\right\}\right)^{d} \\
& \cdot\left(M_{G}\left\{-\sum_{l=1}^{k}\left[\exp \left(x_{l}^{\prime} \beta\right) \cdot \Lambda_{l}\right]\right\}\right)^{1-d},
\end{aligned}
$$

where $M_{G}\{\cdot\}$ is recognized as the moment generating function corresponding to the unobserved heterogeneity distribution $G$.

In our empirical work, we consider both discrete and continuous unobserved heterogeneity distributions $G$. In the continuous case, we consider the scaled Gamma distribution $G(d v \mid x, \theta)=g(v \mid \theta) d v$ with unit mean (for identification) and $\operatorname{var}(v)=\sigma^{2}$, i.e., the density is

$$
g(v)=\frac{v^{\sigma^{-2}-1}}{\left(\sigma^{2}\right)^{\sigma^{-2}} \Gamma\left(\sigma^{-2}\right)} e^{-v \sigma^{-2}}, \quad v>0 .
$$


It follows that the moment generating function $M_{G}\{x\}=E_{g} e^{v x}$ is given by

$$
M_{G}\{x\}=\left(1-x \sigma^{2}\right)^{-\sigma^{-2}} .
$$

Inserting this in (20), we get the log likelihood for $n$ individuals, to be compared to (10),

$$
\begin{aligned}
l(\theta)= & \sum_{i=1}^{n} \ln \left\{\begin{array}{c}
d_{i}\left[\left(1+\sigma^{2} \sum_{l=1}^{k_{i}-1}\left[\exp \left(x_{i, l}^{\prime} \beta\right) \cdot \Lambda_{l}\right]\right)^{-\sigma^{-2}}-\left(1+\sigma^{2} \sum_{l=1}^{k_{i}}\left[\exp \left(x_{i, l}^{\prime} \beta\right) \cdot \Lambda_{l}\right]\right)^{-\sigma^{-2}}\right] \\
+\left(1-d_{i}\right)\left(1+\sigma^{2} \sum_{l=1}^{k_{i}}\left[\exp \left(x_{i, l}^{\prime} \beta\right) \cdot \Lambda_{l}\right]\right)^{-\sigma^{-2}}
\end{array}\right\} \\
= & \sum_{i=1}^{n} \ln \left\{\begin{array}{c}
d_{i}\left(1+\sigma^{2} \sum_{l=1}^{k_{i}-1}\left[\exp \left(x_{i, l}^{\prime} \beta\right) \cdot \Lambda_{l}\right]\right)^{-\sigma^{-2}} \\
+\left(1-2 d_{i}\right)\left(1+\sigma^{2} \sum_{l=1}^{k_{i}}\left[\exp \left(x_{i, l}^{\prime} \beta\right) \cdot \Lambda_{l}\right]\right)^{-\sigma^{-2}}
\end{array}\right\}
\end{aligned}
$$

A slightly different log likelihood function was derived by Meyer (1990) who only attributed a duration $k_{i}-1$ to right censored individuals, instead of $k_{i}$ as with our definitions. ${ }^{6}$ The parameter vector is $\theta=\left(\beta, \sigma^{2}, \Lambda_{1}, \ldots, \Lambda_{K}\right)$ in the nonparametric baseline case, $\theta=\left(\beta, \sigma^{2}, \gamma\right)$ with no baseline duration dependence, and $\theta=\left(\beta, \sigma^{2}, \gamma, \alpha\right)$ with Weibull baseline.

The continuous unobserved heterogeneity approach has been criticized by Heckman and Singer (1984a,b), who argue that it over-parametrizes the model, and emphasize that an incorrect choice of $G$ leads to inconsistent estimates of the parameters of interest. Instead, they suggest a non-parametric maximum likelihood estimate of the mixing distribution, based on the results of Lindsay (1983a,b). The idea is to model $v$ as having a discrete distribution, written as $\operatorname{Pr}\left(V=v_{j}\right)=p_{j}$, with $J$ support points $v_{j}$ and associated probabilities $p_{j}, j=1, \ldots ., J$. Using this discrete distribution for $G$ in (19) leads to the log likelihood function

$$
l(\theta)=\sum_{i=1}^{n} \ln \left\{\begin{array}{c}
\sum_{j=1}^{J}\left(1-\exp \left[-v_{j} \cdot \exp \left(x_{i, k_{i}}^{\prime} \beta\right) \cdot \Lambda_{k_{i}}\right]\right)^{d_{i}} \exp \left[-v_{j} \cdot \exp \left(x_{i, k_{i}}^{\prime} \beta\right) \cdot \Lambda_{k_{i}}\right]^{1-d_{i}} \\
\cdot \prod_{l=1}^{k_{i}-1} \exp \left[-v_{j} \cdot \exp \left(x_{i, l}^{\prime} \beta\right) \cdot \Lambda_{l}\right] p_{j}
\end{array}\right\}
$$

The parameter vector is now be estimated by maximum likelihood, subject to $v_{1}=1$ (a normalization, for identification) and $p_{1}=1-\sum_{j=2}^{J} p_{j}$. Thus, $\theta=\left(\beta, v_{2}, \ldots, v_{J}, p_{2}, \ldots, p_{J}, \Lambda_{1}, \ldots, \Lambda_{K}\right)$ with nonparametric baseline, $\theta=\left(\beta, v_{2}, \ldots, v_{J}, p_{2}, \ldots, p_{J}, \gamma\right)$ without baseline duration dependence, and $\theta=\left(\beta, v_{2}, \ldots, v_{J}, p_{2}, \ldots, p_{J}, \gamma, \alpha\right)$ with Weibull baseline.

Since we are interested in retirement behavior of older individuals, we follow all workers in our sample from the year where they turn 50, which amounts to stock sampling. Following Heckman and Singer (1984a,b), those with high hazard in the full population may have already retired before age 50, and so are underrepresented in the sample. However, our approach does identify the conditional distribution, given workers are still working at age 50. Early exits before age 50 are events rather different from the regular retirement behavior we want to study. Flow sampling is not an alternative, since by our definitions the retirement state is absorbing.

\footnotetext{
${ }^{6}$ With the alternative definition, the coefficients $d_{i}$ and $1-2 d_{i}$ in $(23)$ are replaced by 1 and $-d_{i}$, respectively. Equivalent likelihood values to ours could be obtained by including the first year after censoring in the measured durations under the alternative definition.
} 


\subsection{Empirical Results}

Results of maximum likelihood estimation on the full sample are shown in Table 3. Panel A shows results for labor market, education, occupational, marital status, gender, and regional variables. Results for financial and health variables are shown in Panel B. The first two columns are for the case without baseline duration dependence. Numbers in the first column are parameter estimates. Asymptotic standard errors are given in the second column. Based on these, coefficients that are significantly different from zero at the $5 \%$ level are set in bold face in the first column, and coefficients significant at the $10 \%$ level are in italics. Results for Weibull and nonparametric baseline specifications are reported in a similar fashion in the remaning four columns of the table.

\subsubsection{No Baseline Duration Dependence}

From the first line in the first column of Table 3 (exponential baseline hazard, equation (13)), married individuals tend to retire later (negative coefficient, i.e., lower retirement hazard) than singles in these data. This is perhaps surprising and not in accordance with results in the literature (e.g., Hurd (1990)). We return to this point later. From the next line, males continue working longer than females, which accords better with the literature (e.g., Antolin and Scarpetta (1998), Heyma (2004)). Living in the Copenhagen metropolitan area decreases the retirement hazard, consistent with the finding of An, Christensen and Datta Gupta (2004) who also use Danish data and attribute the effect to higher housing prices in the capital.

Labor Market Variables The next group of variables in Table 3 relate to the labor market. Working full time is associated with stronger attachment to the labor market. The retirement hazard is about half of that of persons not working full time. Retirement rates increase with the unemployment rate, consistent, e.g., with Lindeboom (1998). This effect may be understood by recalling that a portion of the individuals at risk are unemployed and seeking employment. Higher unemployment rates may be associated with increased layoff rates combined with decreased job-finding opportunities. In addition, the effect may be related to the government's attempts to reduce youth unemployment by providing incentives for the older generation to retire.

Greater labor market experience is associated with a higher retirement hazard. The magnitude of the effect is considerable. As experience in these data runs from 0 to 21 years and is scaled to between 0 and 1 , the estimated coefficient indicates that an additional year of experience increases the hazard by about $15 \%$. Individuals who have participated over a long period have had more years to build up retirement savings, have possibly contributed to a pension fund, and may be more likely to meet eligibility criteria. In particular, a requirement for early retirement (the labor market based post employment wage route) is membership of a UI-fund for 20-25 years, and membership can only be maintained for a limited period if the individual is unemployed. This helps explain the increase in retirement hazard associated with both higher experience and UI-fund membership (the next variable in the Labor Market group in the table). We return to this point when separating out the early retirement route in a multivariate model below.

A worker who has received sickness benefits during the year is more likely to retire. This seems reasonable, 
since poor health increases the individual's uncertainty about the future in the labor market, and also may lower human capital as in the case of a long unemployment spell, thus increasing the probability of leaving employment (see, e.g., Diamond and Hausman (1984), Antolin and Scarpetta (1998)). Including the disease indicators based on objective diagosis codes as additional regressors in Panel B below allows deriving a more detailed picture of the effect of health on retirement behavior.

Education Overall, we find that a higher level of education is associated with later retirement. The reference group is individuals with basic education (no education beyond primary school). The finding is in agreement with the empirical literature, e.g., Diamond and Hausman (1984). People with higher education may have a lower replacement rate if entering a retirement scheme. Furthermore, this group has had a delayed entry into the labor market. The estimated effect is not monotonous in education level, i.e., medium duration higher education is associated with higher retirement rate than short duration higher education. In this relation, note that Lindeboom (1998) actually finds a positive effect of high education on the retirement rate.

Occupation The reference group is high-salary workers, and compared to this, workers in other occupations exhibit higher propensities to retire. This is consistent with the empirical results in Heyma (2004), as well as with human capital theory. A person in a high-salary job is likely to have invested a lot in his or her career and is therefore less likely to move to non-employment, see Fields and Mitchell (1984). Low-salary workers exhibit higher retirement rates than medium-salary workers. The positive coefficient for the self-employed is not necessarily in line with the literature, where most authors find a lower retirement rate for this group, see, e.g., Antolin and Scarpetta (1998), but it is the lowest of the coefficients in the Occupation group, i.e., only high-salary workers retire later than the self-employed.

Financial Variables Turning to Panel B of Table 3, individuals with higher income are less likely to retire, consistent, e.g., with Diamond and Hausman (1984). The substitution effect dominates the income effect. Leisure is relatively more expensive for the highly paid. This group might also have saved more, thus increasing the incentive to retire, but this effect is too weak to match the substitution effect, based on our estimation. The weak effect of savings is confirmed by the insignificant coefficient on wealth. The coefficient on own income is stronger than that on household income, perhaps due to better data (lower measurement error). From the point estimate, since the financial variables enter logarithmically, a $10 \%$ increase in own income nearly cuts the retirement probability in half. In a broad sense, that working longer is associated with higher income is consistent with the findings above that it is also associated with longer education, higher occupation, and working full time. Thus, overall, the results for the explanatory variables considered so far make sense and suggest that there is nothing unusual in our sample, compared to the literature.

Health We now consider the objective diagnosis codes that are the explanatory variables of main interest, and that are unique to this study. From Table 3, Panel B, first column, we get several significant effects of these health events on retirement. Perhaps surprisingly, the first result is that being diagnosed with malignant cancer 
during the year lowers the retirement hazard. The way to understand this result is that by construction, the coefficient measures the effect of both obtaining the malignant cancer diagnosis and surviving through the year. Non-survivors are censored. Survivors who get well during the year may want to work longer to compensate for lost work. Surviving malignant cancer may even generate a psychological boost encouraging continued work. Survivors who remain ill by the end of the year may have put labor market decisions temporarily on hold. The corresponding coefficient for benign cancer is insignificant.

We find that retirement hazards are increased by mental and behavioral disorders, diseases of the nervous, circulatory, respiratory, and musculoskeletal systems, and by endocrine, nutritional, and metabolic diseases. Retirement hazards are not significantly impacted by diseases of the digestive or genitourinary systems, or by injury or other diseases, according to this categorization. The highest coefficient and $t$-statistic $(t=5.98)$ are associated with diseases of the musculoskeletal system and connective tissue, which include arthritis and rheumatism. An individual obtaining a diagnosis in this category during the year experiences a $50 \%$ increase in retirement probability. The other significant coefficients imply increases of about $30 \%$ to $40 \%$ in retirement rates for these diseases. Besides malignant cancer, none of the other disease groups gets a significantly negative coefficient.

The last results in the table show that more days of treatment and more hospital admissions during the year are associated with higher retirement hazard, as expected, broadly consistent with the positive effect of having received sickness pay in Panel A. Persons receiving many diagnoses actually retire less, which is perhaps surprising, and may suggest heterogeneity in behavior across the population, with some individuals obtaining more diagnoses without being work incapacitated.

The results confirm the empirical relevance of using objective diagnosis codes as explanatory variables in retirement analysis, and reinforce the notion that changes in health impact labor market behavior. In particular, the diagnosis codes capture a multidimensional force with different effects for different conditions, even when controlling for a number of other health related variables, such as sickness benefits, duration of hospital treatment, number of admissions, etc.. Presumably, as the diagnosis codes are objectively measured, as opposed to self-reports, they should be precisely measured and should not be subject to endogeneity problems. Consequently, the estimated coefficients should be reliable, and, in particular, should not suffer from attenuation and/or endogeneity bias. Thus, the project of adding these variables to the retirement analysis is so far successful.

In the following, we examine whether these findings hold up as we generalize the estimation technology along a number of dimensions. In particular, we consider in turn more flexible duration dependence, unobserved heterogeneity, seperate estimation by gender, and a multivariate model for distinct exit routes.

\subsubsection{Duration Dependence}

Weibull Baseline Results from estimation with the Weibull baseline hazard (15) appear in the second and third columns of Table 3. For most explanatory variables, both point estimates and asymptotic standard errors are very similar to those obtained without accounting for baseline duration dependence above, with a 
few exceptions. First, in Panel A, the very large magnitude of the experience effect without baseline duration dependence is more moderate in the Weibull case. Secondly, self-employed workers now exhibit a lower retirement hazard than all other occupational groups, including high-salaried workers. The estimated coefficient for self-employment is negative and significant at conventional levels, with a $t$-statistic of -9.1 . This is better in line with the literature, e.g., Antolin and Scarpetta (1998), than the result obtained using exponential baseline. Third, from Panel B, greater wealth now increases the retirement hazard. From the point estimate, a doubling of wealth increases the retirement hazard by about $9 \%$. The associated $t$-statistic is 2.8 . This is interesting from a number of perspectives. For one, wealth effects are often difficult to detect due to poor data quality (measurement error in wealth). Secondly, the opposite signs on income and wealth make sense as substitution and income effects. The results on experience, self-employment, and wealth suggest that allowing for baseline duration dependence is empirically relevant and produces meaningful changes in estimates. Indeed, the fourth line of Panel A shows that the logarithm of the Weibull shape parameter, at .63, is greater than zero (the exponential case), and the result is significant $(t=3.2)$. Positive duration dependence makes sense in the context, i.e., all else equal, the individual becomes more likely to retire as time progresses.

The findings on the objective diagnoses are very similar in the constant and Weibull baseline cases, both in terms of point estimates and significance. In particular, seven separate groups of diseases have distinct significant impacts on the retirement hazards, with survival of malignant cancer significantly decreasing the retirement rate. Given the meaningful changes in inference for the other variables when moving to the Weibull model, this serves as a useful robustness check on the estimated effects of health.

Nonparametric Baseline The last two columns of Table 3 show the results using a nonparametric baseline, treating the $\Lambda_{k}$ in (12) as separate parameters, instead of the constant or Weibull parametric specifications of the previous two models. The number of person-year observations is now slightly less, for numerical reasons (see Section 2). From Panel B, the same seven disease categories remain significant under nonparametric baseline, and retain the same signs and orders of magnitude as in the foregoing models. In addition, two more groups now turn significant. Thus, an increase in retirement hazard is associated with benign cancer as well as with injury, poisoning, and other external causes. These diagnosis codes replace number of diagnoses and admissions as significant regressors. As this model is more flexible and less likely to be misspecified, the results show that objective diagnosis codes indeed provide useful means of capturing the effects of health shocks on retirement behavior.

From Panel A, estimation of the nonparametric baseline does come at the cost of losing significance of marital status, as well as medium and long education. Furthermore, working full time is now only significant at the $10 \%$ level. Own income and household income now get opposite signs, consistent with the notion that the substitution effect dominates for own income, whereas household income (including in particular spouse's income) is treated more like wealth, i.e., the income effect dominates, and subsumes the effect of wealth itself that was significant with Weibull baseline.

Figure 2 shows the estimated exponential, Weibull, and nonparametric baseline hazards. Although the nonparametric hazard is nearly monotonically increasing, consistent with the Weibull estimate, there is a local 
peak at 60 and a trough at 62 , coinciding with those in the Kaplan-Meier hazard in Figure 1. Furthermore, we see that the slope of the nonparametric hazard is steeper than the Weibull. This suggests that the nonparametric specification is an improvement.

\subsubsection{Unobserved Heterogeneity}

Table 4 shows the results from estimation allowing for unobserved heterogeneity, as represented by $v$ in (17). We consider first a parametric approach, with gamma unobserved heterogeneity as in (21), and either constant or Weibull baseline hazard, using (14) respectively (16) for $\Lambda_{l}$ in the log likelihood (23), then a nonparametric approach with discrete unobserved heterogeneity (using $J=2$ support points) combined with nonparametric baseline hazard, i.e., treating $\Lambda_{k}$ as separate parameters in (24).

From Panel A, there is now significantly less retirement among the married across all three baseline hazard specifictions, including the nonparametric that produced an insignificant result for this variable without unobserved heterogeneity (Table 3). Similarly, the effect of long eduation is now significantly negative throughout, as well, even though with nonparametric baseline the point estimate (at -.21) is smaller in magnitude than for short education, and medium education remains insignificant. In the Weibull model, the positive duration dependence is much more pronounced when allowing for unobserved heterogeneity. The point estimate of the Weibull parameter in Table 4 is more than twice that in Table 3, and the standard error drops to less than half. Also in the Weibull model, the apparent negative effect of self-employment drops out, leaving inference closer to that with other baseline specifications.

Interestingly, from Panel B of Table 4, the result that benign cancer significantly increases the retirement hazard once the nonparametric baseline is allowed for carries over to the case with unobserved heterogeneity. All other qualitative results on diseases and other explanatory variables agree with those from the previous table. As there is least risk of misspecification when allowing for both unobserved heterogeneity and nonparametric baseline, the cases where inferences from this specification (last two columns of Table 4) agree with those from simpler specifications are relatively reliable. Thus, surviving malignant cancer indeed seems to reduce subsequent retirement. In other cases, inferences from the most general models are probably preferred, so by this criterion the effect of married status appears to be to reduce retirement, and there is even an indication that more wealth may in fact reduce retirement (significance at $5 \%$ in the parametric case and $10 \%$ in the nonparametric in Table 4).

All significant coefficients on diagnosis codes in the the most general model so far (last two columns of Table 4, Panel B) are equal to (up to two digits) or greater in magnitude than the corresponding point estimates in Table 3, Panel B. Ignoring unobserved heterogeneity will always lead to a downward bias in the estimated hazard due to attrition (Heckman and Singer (1984a,b)), and the associated attenuation bias on individual coefficients is toward zero. Our results are consistent with this, and thus suggest that unobserved heterogeneity is present. Indeed, from the bottom portion of Table 4, Panel B, the gamma mixture distribution parameter for the exponential and Weibull models and the discrete heterogeneity parameters for the nonparametric specification are all statistically significant. The estimated discrete mixture implies that $p_{2}=14 \%$ of the sample has $v_{2}=1.4$, significantly 
greater than unity. Thus, the results strongly suggest that baseline hazard and homogeneity restrictions that may imply misspecification should be relaxed.

Figure 3 shows the effect on the estimated nonparametric baseline hazard of including unobserved heterogeneity. The dashed line represents the estimated hazard in the model with unobserved heterogeneity, whereas the solid line is that without, repeated from Figure 2. Introducing unobserved heterogeneity allows the baseline hazard to pick up stronger positive duration dependence, consistent with the difference in the Weibull parameter estimates with and without unobserved heterogeneity (1.39 versus 0.63 ). The nonparametric estimation shows that the difference is largest at earlier ages (shorter durations) consistent with the initial sample being more mixed, whereas for long durations individuals with better (and thus less dispersed) unobserved characteristics tend to dominate.

On the substantive side, the results point to an important role for objectively measured health variables for retirement behavior, even when controlling for labor market and socio-economic background variables.

\subsubsection{Gender Differences}

We continue with the general specification allowing for nonparametric baseline hazard as well as unobserved heterogeneity (as in the last two columns of Table 4) and fit this separately to the male and female subsamples to examine whether there are gender differences in the effects of diseases and other explanatory variables on retirement behavior. Small sample size has often forced researchers to control for gender only by including a gender dummy among other regressors. However, some of the earlier retirement studies not using diagnois codes (e.g., Lindeboom (1998), An, Christensen, and Datta Gupta (2004)) suggest that there may be gender differences in several coefficients.

Table 5 shows the results from separate application of the model to the male and female subsamples. From the first line of the table, we now find that the significant reduction in retirement hazard associated with married status, common to all the previous analyses, only remains in the male subsample. In the female subsample, the effect is reversed. The retirement hazard is significantly higher for married women than for the unmarried. The point estimate for the full sample, at -.08 in Table 4 , is in between the two subsample estimates in Table 5 ( -.29 for males versus .14 for females), and this is so for all the variables in Panel A of the two tables, which makes sense. One interpretation of the result for marital status is that married women can better afford to retire, as they are provided for by their working husbands. The husbands work longer to compensate for the loss of wife's earnings, and the male retirement hazard is strongly decreased, given married status. From the point estimates, being married reduces the retirement hazard for males about as much as having short or long higher education, as opposed to basic or vocational training. For females, married status increases the retirement hazard by as much as being member of a UI-fund.

The city variable, which was before significantly negative (lower retirement rates in the capital), is now only so for women. Working full time reduces the hazard for men, consistent with the findings for the full sample, whereas the effect is insignificant for women. The remaining labor market variables have the same effects as in the foregoing analysis, except that experience loses its significance among men. 
In the previous estimations, the vocational, short, medium, and long education variables generally got negative coefficients (recall that the left out category is basic education), but significance of the effect was lost for medium length education in the most general model with nonparametric baseline, cf. Table 4 . From the separate estimation by gender, the explanation is seen to by that medium length education in fact gets a significantly positive coefficient for women. The corresponding male coefficient is negative, albeit insignificant. In Denmark, the medium length educations pursued by women typically lead to carreers as nurse, school teacher, social worker, or similar jobs that are not highly paid relative to the educational requirements, but are nevertheless demanding for older workers. Medium length educations pursued by larger fractions of men include engineer, accountant, army officer, etc., and this may explain the gender difference in the result.

The effects of the occupation indicators do not change much compared to previous estimations and are similar across gender, except that the positive effect (earlier retirement) of being an assisting spouse now is only significant among women. Since husbands are older than wives on average, it is plausible that women working as assisting spouses retire at the same time as their husbands, and if the husband selects a normal retirement age, this makes the wife's retirement age relative low, thus explaning the positive effect among females. On the other hand, few husbands work as assisting spouses, and those who do will not exhibit a particularly low retirement age even if they retire jointly with their wives who on average are younger.

Turning to the financial and health variables in Panel B, the negative effect on the retirement hazard of own income from the full sample in Table 4 carries over to each subsample in Table 5 . The result that the substitution effect dominates the income effect for both men and women is consistent with Blau (1997), using U.S. data, and An, Christensen, and Datta Gupta (2004), using data from Statistics Denmark. The opposite coefficient on household income is now no longer significant for men, but much stronger for women than in the full sample, consistent with the notion that particularly women treat household income (especially husband's income) as wealth in retirement considerations. The result that the positive cross-effect of income is strongest for females is consistent with Blau (1997).

Our finding that surviving malignant cancer reduces the retirement hazard carries over to both the male and the female subsample. The coefficient was -.66 in the full sample, with a $t$-statistic of -5.67 , and again, this full sample point estimate is about halfway between the male and female subsample estimates. The reduction in hazard following malignant cancer is found to be strongest in the male subsample, with a coefficient of -1.26 and a $t$-statistic of -6.40 , whereas the coefficient in the female subsample is -.28 , for a $t$-statistic of -1.80 . Apparently, if surviving malignant cancer is associated with a positive psychological stimulus to work more after the incident, then this effect is particularly strong for men. Similarly, the effect that benign cancer in fact induces retirement (as seen in the full sample) is in fact specific to men, and insignificant for women. The effect of endocrine, nutritional, and metabolic diseases is now only significant at a 10 percent level for men and insignificant for women, whereas it was significantly positive in the full sample. The increase in hazard is stronger for women and weaker for men than in the full sample in case of diseases of the nervous, circulatory, respiratory, and musculoskeletal systems. The greatest gender differences are seen in the effects of diseases of the circulatory and musculoskeletal systems, with women responding with earlier retirement than men in 
these cases. On the contrary, the increase in retirement hazard associated with mental and behavioral disorders is stronger among males than among females. The effect of diseases of the genitourinary system, which was insignificant in the full sample, now becomes significant at the 10 percent level, with a positive coefficient for women and perhaps surprisingly a negative coefficient for men. Significance of injury, poisoning and other consequences of external causes is lost when separating the sample by gender. Diseases of the digestive system, number of diagnoses, and number of admissions remain insignificant in the separate subsamples, as in the full sample. The effect of days of treatment is significantly stronger for women than for men. The estimates of the unobserved heterogeneity distribution suggest that the types have been switched in the subsamples, but that for both subsamples, the mixing probability is significantly different from both zero and unity, showing that accounting for unobserved heterogeneity remains important even after separating the sample by gender.

Overall, the results confirm the feasibility of separate estimation by gender of the general model. Indeed estimated coefficients do differ by gender. This is not due to a difference in estimated baselines, which are similar across gender, see Figure 4, and close to that for the full sample in Figure 2.

\subsection{Grouped Duration Competing Risk Model}

In this section we consider a competing risk model that separates the alternative retirement routes. This approach is useful in assessing the importance of health, financial and other explanatory variables for retirement behavior, since the impact may differ by program. Our implementation uses five distinct exit routes from the labor market, with a separate nonparametric baseline hazard for each. This specification is already very general, and for parsimony we again control for gender through a dummy rather than by the sample separation method from Section 3.2.4. We do not consider unobserved heterogeneity (it was treated in Sections 3.2.3 and 3.2.4), although it might have been considered as an alternative to the five separate nonparametric baselines. It would be hard to identify both unobserved heterogeneity and nonparametric baselines jointly, due to the dimensionality in the competing risk case, and there is some specific interest in the differences between baselines corresponding to separate labor market exit routes. ${ }^{7}$ The five routes considered are disability, early retirement, unemployment followed by early retirement, unemployment followed by exit routes other than early retirement, and, finally, all other programs (e.g., transition pay, civil service pension, social assistance, etc.). Here, early retirement is a labor market pension scheme (see the appendix for further details on the different retirement programs available).

\footnotetext{
${ }^{7}$ Based on the analytical and simulation results in Ridder (1987), flexible baselines are more important for the regression parameters than correct heterogeneity specification.
} 


\subsubsection{Likelihood function - Competing risk}

Following the seminal work of Han and Hausman (1990) and Sueyoshi (1992), in the competing risk framework the probability (8) of surviving the $k^{\prime}$ th interval given survival through the first $k-1$ is generalized to

$$
\begin{aligned}
P\left(T>t_{k} \mid T>t_{k-1}, x_{k}\right) & =\exp \left[-\sum_{r=1}^{R} \int_{t_{k-1}}^{t_{k}} h_{r}\left(t \mid x_{k}\right) d t\right] \\
& =\prod_{r=1}^{R} \alpha_{k}^{r}\left(x_{k}, \theta\right),
\end{aligned}
$$

where $R$ is the number of competing risks, $h_{r}\left(t \mid x_{k}\right)$ is the hazard for exit via route $r$, and

$$
\alpha_{k}^{r}\left(x_{k}, \theta\right)=\exp \left[-\int_{t_{k-1}}^{t_{k}} h_{r}\left(t \mid x_{k}\right) d t\right] .
$$

Under the assumption of proportional hazard for each risk, the generalization of (11) is

$$
h_{r}\left(t \mid x_{k}\right)=\lambda^{r}(t) \exp \left(x_{k}^{\prime} \beta^{r}\right)
$$

where $\beta^{r}$ denotes the destination-specific sensitivities to explanatory variables, and in this case we have

$$
\alpha_{k}^{r}\left(x_{k}, \theta\right)=\exp \left[-\exp \left(x_{k}^{\prime} \beta^{r}\right) \Lambda_{k}^{r}\right]
$$

in (25), with $\Lambda_{k}^{r}=\int_{t_{k-1}}^{t_{k}} \lambda^{r}(t) d t$ the $r^{\prime}$ th integrated hazard. In the empirical application, $R=5$. The probability of an exit to state $r$ in interval $k$ is $1-\alpha_{k}^{r}$, and the total retirement probability is $1-\prod_{r=1}^{R} \alpha_{k}^{r}$.

The contribution to likelihood for an individual with duration $k$ and destination state indicators $d^{r}, r=$ $1, \ldots, R$ is

$$
L(\theta, k, x)=\left[\prod_{r=1}^{R}\left(1-\alpha_{k}^{r}\left(x_{k}, \theta\right)\right) \prod_{j=1}^{k-1} \prod_{\ell=1}^{R} \alpha_{j}^{\ell}\left(x_{j}, \theta\right)\right]^{d}\left[\prod_{j=1}^{k} \prod_{r=1}^{R} \alpha_{j}^{r}\left(x_{j}, \theta\right)\right]^{1-d}
$$

where the right-censoring indicator is $1-d=1-\sum_{r=1}^{R} d^{r}$.

The log likelihood function for a sample of $n$ individuals is

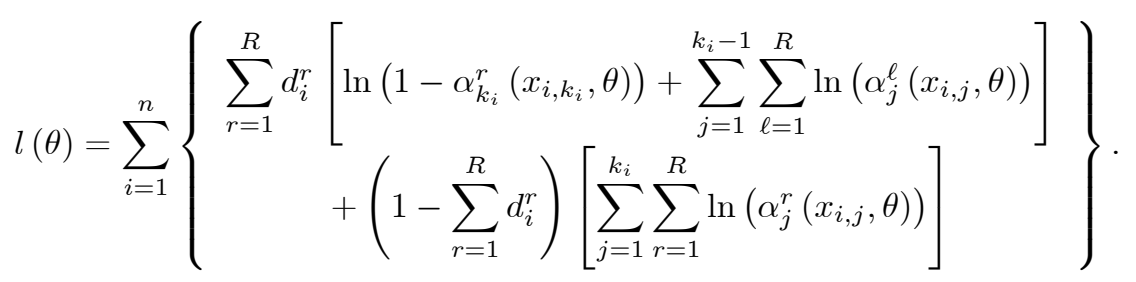

This is maximized numerically with respect to the parameter vector $\theta=\left(\beta^{1}, \ldots, \beta^{R}, \Lambda_{1}^{1}, \ldots, \Lambda_{K}^{1}, \Lambda_{1}^{2}, \ldots, \Lambda_{K}^{R}\right)$, again using BFGS.

\subsubsection{Results - Competing Risk}

Table 6 summarizes the duration data for each of the five destination states, in the same manner as Table 1 for the full sample. The eligibility age for the early retirement program is 60 , and with workers followed from age 50 , the minimum observed duration is 10 . Upon reaching eligibility, most of those exiting through this program 
do so within the first few years. By comparison, exit through the disability route is much more spread out across all ages, as the medical requirements for this program may be met at any age. In fact, there are more exits through this route before reaching early retirement eligibility (duration 10) than after.

The third destination, unemployment followed by early retirement, shows exits (into unemployment) largely concentrated before duration 10 (early retirement eligibility), as workers becoming unemployed after reaching this age have the option of transiting directly to early retirement rather than spending time in the unemployment program, conditionally on having paid into a UI-fund for sufficiently long. Most of the exits before duration 10 (age 60) are at durations 7 through 9 (ages 57 through 59), and this effect is possibly explained by the tendency on the part of firms to lay off older workers during this period. The effect is not explained by the maximum duration of unemployment insurance benefits, which is four years in Denmark, since this maximum does not apply to workers between ages 55 and 59 who will become eligible for early retirement by age 60 (having paid into the system for the required number of years). In this manner, benefits could extend up to nine years, thus explaining the earlier exits through this route, starting at duration 1 (two observations).

The fourth destination, unemployment followed by other exit routes than early retirement (fourth column in Table 6), shows exits more spread out than unemployment followed by early retirement, since some of the retirement programs following the unemployment spell may commence before age 60, e.g., civil service pension and disability. Finally, the residually determined destination labelled Others in the table is even more spread out, and this includes exits into civil service pension and other schemes not preceded by an unemployments spell, so no particular age or spell duration requirement applies.

Comparing with Table 1, the similar full sample durations, the durations in Table 6 clearly suggest that disaggregating the hazards by the heterogenous destination states is relevant. The following analysis examines whether differences by destination states arise in the effects of health, financial and other explanatory variables, as well.

Figure 5 shows the estimated baseline hazards for the five separate exit routes. The individual coefficient estimates are discussed below. From Figure 5 we see that disability has the highest baseline throughout. Unemployment followed by early retirement starts out lowest, but exhibits more positive duration dependence, and nice continuity with the estimated baseline for early retirement when this program becomes available. Unemployment followed by other programs has a baseline in between those for the disability and unemployment followed by early retirement routes.

Disability versus Early Retirement A person can receive disability pay if he or she is permanently unable to work and does not receive any other type of pension. It is required that all possibilities to improve the applicant's labor market qualifications concerning rehabilitation, treatment, active social policy, etc. have been tried. On the other hand, early retirement pay (post employment wages) may be received independently of any medical criteria.

Results for exit to disability appear in Table 7, columns 1 and 2, and results for early retirement in columns 3 and 4. The insignificant result for marital status from the corresponding model with nonparametric baseline in the last two columns of Table 3 is now replaced by a finding that being married increases the tendency to retire 
via the early retirement route and decreases the tendency to retire via the disability route. This underscores the relevance of the competing risk specification, i.e., lumping states with opposite characteristics may just result in insignificant effects in a single risk estimation. Presumably, early retirement may in part be chosen in order to increase leisure time, and among married individuals this increased leisure time may be shared with the spouse, so the result is consistent with complementarities in leisure times. On the other hand, if married couples in this manner engage in joint retirement planning, they may not be able to select the disability route, since this requires that specific medical criteria be met. The result suggests that a person whose health deteriorates sufficiently to qualify for disability may nevertheless postpone this decision if he or she is married. This may be due to either of two factors. The first is the possibility of coordinating retirement with the spouse later. The second is the possibility that the spouse is able to care for the individual in poor health even though he or she meets criteria for disability. It is noted that the negative effect of marriage using constant or Weibull baseline (Table 3) seems to be driven by disability rather than early retirement, hence showing that separating by destination is more informative.

The significant lowering of the retirement hazard when living in the city seen in the previous results is driven only by early retirement, whereas the coefficient is insignificant for disability and indeed positive for the other programs in the table. This is consistent with the notion that early retirement is the route with strongest voluntary choice element, whereas some workers may be forced into disability for medical reasons. Similarly, the aggregate results for the labor market attachment variables are seen to be driven by early retirement, e.g., the positive effect of experience and UI-fund membership carry over and the magnitude of the coefficients is strongly increased. This is as expected, since these variables enter the eligibility criteria for the early retirement program, whereas both variables decrease the rate of disability retirement. Those with strong labor market attachment choose early retirement over disability. Also, having had a larger degree of unemployment during the year increases the exit rate via early retirement, not via the disability route. On the other hand, the previous positive result on the sickness benefit variable is seen to be driven by the disability destination, consistent with this being health related, whereas the variable has no impact on early retirement.

Again, we find a significantly negative effect from the length of education, and here, signs and significance are more similar across disability and early retirement. The negative point estimates (the left out group is basic education) are larger for disability than for early retirement, consistent with evidence that individuals with higher education tend to have higher life expectancy and better health outlook due to better cognitive ability and decision-making. Other factors that improve with increased educational attainment, such as income, occupational safety, and access to health insurance, are also associated with better health outcomes (Wong, Shapiro et al., 2002; Muennig et al 2005), which should in turn lower disability retirement. In fact, the results on education are now stronger than in the single-risk case (Table 3), both in terms of magnitude of point estimates and in that also long education gets a significantly negative coefficient, and this is so for both disability and early retirement.

From Panel B, the previous insignificant result for wealth is now replaced by an increase in early retirement among the wealthy, who can afford this voluntary retirement and hence increase in leisure time, along with 
a decrease in disability retirement among the more wealthy. An earlier finding of a negative effect of wealth in Danish data was by Pedersen and Smith (1991) who used a single-risk analysis with Weibull baseline. Our results suggest that the negative effect is driven by disability retirement. Possibly health is better among the wealthy, who then do not to the same extent require disability.

Turning to the effects of the health variables, the reduction in retirement after surviving malignant cancer carries over to both disability and early retirement, and also unemployment (the next four columns in the table). It is clear that most of the other significant results from Table 3 are driven by exit through the disability route, which makes sense, since this is more health related than early retirement. Even for disability, endocrine, nutritional, and metabolic diseases, as well as diseases of the nervous system and sensory organs, drop to significance only at a $10 \%$ level, possibly due to sample size. Diseases of the genitourinary system decrease the probability of entering disability, whereas it was insignificant before. Comparing across programs in Table 7, it is seen that this type of disease may lead to unemployment. This is interesting, since these diseases typically will not qualify an applicant for disability, yet they may apparantly influence a person's ability to work, based on these results. Further, the disability results confirm the significance of the two additional disease categories that appeared when moving from parametric to nonparametric baseline in Table 3, namely, benign cancer and injury, poisoning, etc. An increase in the number of diagnoses decreases the hazard rate into disability, whereas the number of admissions increases it, just as in Table 3 with constant or Weibull baseline.

The effect of health variables on early retirement are mostly significant only at $10 \%$ or higher, confirming that health problems induce retirement through the disability route where specific health criteria must be met, rather than retirement via the voluntary labor market based early retirement route. Indeed, only diseases of the nervous, circulatory, and genitourinary systems remain significant for early retirement, and only at the $10 \%$ level. For the first category, the nervous system, the coefficient is larger for early retirement, at .39, than for disability, at .25. The pattern is reversed for the second category, diseases of the circulatory system, where the coefficient is larger for disability, at 1.14, than for early retirement, .23. In our data, we do not have disability or early retirement eligibility dummies, but the nonparametric baseline hazard controls for age, and hence in particular for being in the early retirement eligibility age range (starting at 60 in our sample period). In addition, we do control for whether workers are members of a UI-fund, a further early retirement eligibility criterion. The results show that there are differences between the types of disease with respect to the rates of retirement into different programs. Those hit by diseases of the nervous system and sensory organs tend to exit via the early retirement route, and those suffering from diseases of the circulatory system via the disability route. It is possible that the health related criteria for disability retirement are more easily satisfied in case of diseases of the circulatory system, whereas those who have worked sufficiently and paid into the early retirement program along the way are more likely to develop diseases of the nervous system and sensory organs, including, e.g., stress related illnesses. Needless to say, these types of results and comparisons are unique to our study using a large register based data set and the competing risk approach.

Unemployment Followed by Alternative Retirement Routes We now take a closer look at individuals for whom unemployment is the last labor market state prior to retirement. They could potentially have been 
planning their exit from the labor market already at the time of becoming unemployed, and hence this analysis. It is especially interesting to distinguish between those whose unemployment spell is followed by voluntary early retirement and those who retire through other routes. In particular, the exception to the general UI-fund membership requirement for members between the ages of 55-59 years makes it likely that the unemployment spells of some have been actually considered as de facto early retirement. Thus, the maximum unemployment duration of four years (before being moved to welfare) is not applicaple during the sample period to those who are 55 years of age or older when hitting the four year barrier, provided they have been paying into the early retirement program and will become eligible for this when reaching age 60 . In effect, this allows for early retirement through the combined unemployment-early retirement route already at the age of 51 . We may look for signs that the combined unemployment-early retirement route shows signs of being more similar to other retirement routes, especially the voluntary early retirement route, than unemployment followed by other retirement programs, where the initial unemployment spell is more likely involuntary.

In Panel A, Table 7, Columns 5 and 6, the male indicator is only significant at 10\%. In the single state case, as well as in all other states in the competing risk model, male is significantly negative at $5 \%$ or better. Furthermore, the point estimate for the combined unemployment-early retirement state is only -.1 , as opposed to -.3 or greater (in magnitude) in all other cases. This suggests that in particular males use unemployment as an early route into retirement, some likely exploiting the exemption from the four-year rule. Married status is insignificant for unemployment followed by early retirement, just as in the single state model with nonparametric baseline (Table 3), but negative for unemployment followed by other programs. The results suggest that married individuals prefer the early retirement exit route, presumably as a means of coordinating early retirement with the spouse to enjoy shared leisure time, consistent with complementarity.

Living in the city increases the rate of unemployment followed by early retirement, and even more so unemployment followed by other programs, whereas this variable had a negative effect on early retirement itself, and on retirement in general in Table 3. This shows that the phenomenon of entering into unemployment, then retirement, is a city phenomenon.

We find a positive and significant effect from working full time, consistent with this group being eligible both for generous unemployment benefits and often in addition attractive early retirement pay, whereas the full time variable had the opposite sign for straight early retirement and disability retirement. These opposing effects may explain the insignificant coefficient in the model with nonparametric baseline in Table 3, underscoring again that the competing risk specification is warranted. The coefficient on full time is smaller for unemployment followed by other programs than for unemployment followed by early retirement, and only significant at $10 \%$, consistent with the attractiveness of not only the UI benefit but also the early retirement portion of the combined route.

The coefficient on the unemployment rate (measured before exiting) is higher for the two unemployment exit routes considered, consistent with the unemployment state being more easily accessible to the individuals exiting through this route.

More experience decreases the probability of exiting via the unemployment state, which makes good sense, in that greater experience should make unemployment less likely in general. Experience also gets a negative 
coefficient in case of the disability route, but a strongly positive (much larger) coefficient for the early retirement route, consistent with the notion that this route depends on previous labor market attachment, and it is seen that it is the latter effect that drives the positive result in Table 3.

Membership of a UI-fund is naturally strongly significant for the two unemployment routes, as for the early retirement route where UI-fund membership is required, as well, whereas this variable gets a negative sign for disability, and the combined result in Table 3 was a small (positive) coefficient. Sickness pay gets a positive coefficient for the unemployment routes, as for disability, whereas there is no effect for the early retirement state, showing again that the latter is less of a health based choice, but that health problems may spur unemployment.

The education variables drop in significance. We only find a negative effect of short education (at 10\%) for unemployment followed by early retirement and a positive effect of long education (again at 10\%) for unemployment followed by other programs.

Turning to Panel B, unemployment followed by early retirement has lower significance of household income and wealth than unemployment followed by other programs. In fact, the latter is similar to disability, and the former to early retirement itself, in that the large coefficient on household income becomes small and less significant, whereas the negative coefficient on wealth turns positive. Thus, unemployment followed by early retirement appears most similar to the voluntary early retirement route itself.

Again, the number of significant health variables drops, compared to disability, but unemployment can result from genitourinary diseases, that had a negative effect on disability and early retirement. Still, several diseases remain significant, in particular malignant cancer (still with a negative coefficient), endocrine, nutritional, and metabolic diseases, diseases of the genitourinary system (previously insignificant), and diseases of the musculoskeletal system and connective tissue.

We conclude that unemployment followed by early retirement is different from unemployment followed by other programs, and in some respects, particularly the effects of financial variables, this combined exit route is more similar to voluntary early retirement itself, i.e., we have found a portion of unemployment that appears planned. Again, this analysis hinges on the competing risk approach.

Other programs Finally, in the last two columns of Table 7, we consider retirement into other programs in general. This is negatively affected if the person is working full time or member of an UI-fund. These findings seem reasonable since the variables are not part of the eligibility criteria for the programs included in the category. The same pattern is seen for disability. Next, the point estimate for the unemployment rate increases, compared to disability, and sickness pay turns insignificant, so the residual state is different. We only find a negative significant effect (at 10\%) for workers with medium education, and among the occupation indicators, low salaried workers is no longer significant. Turning to Panel B, household income and wealth also lose significance. On the other hand, regarding the health variables, there are several significant diagnosis code categories, more in line with the number of significant categories in Table 3. The coefficient on diseases of the digestive system is higher for this exit route than for any other, again confirming that the retirement programs are different, and that the health variables matter for all. 


\section{Conclusion}

People leave the labor force for many different reasons, voluntarily or not, through various arrangements such as unemployment benefits, disability benefits, or special early retirement schemes. This paper contributes to the literature by considering a large, register-based sample including objective medical diagnosis codes. We estimate detailed hazard models of duration until retirement, controlling for unobserved heterogeneity and nonparametric baseline hazards, as well as observed heterogeneity through time-varying explanatory variables. These include diagnosis codes, along with a host of demographic, labor market, and financial regressors. The panel structure of the data allows following individuals year by year from the age of 50 and precisely measure changes in objectively measured health and other regressors, as well as labor market status. We consider 12 broad, mutually exclusive and exhaustive categories of health diagnoses defined by aggregation across ICD codes. The use of objective medical diagnosis codes should eliminate the justification or rationalization bias due to self-reports of health, and the large sample size obtained by using register rather than survey data should mitigate the effect of any remaining mismeasurement of true work incapacity. Together, these improvements should help distinguish empirically important effects of health and economic variables on retirement. We distinguish a number of alternative exit routes, in particular, disability, early retirement, unemployment, and others (including out of the labor force and welfare). In case of the unemployment state, we distinguish between whether the unemployment spell is followed by voluntary early retirement or other programs. We estimate both single risk models, lumping all retirement states, and competing risk specifications, including all separate exit routes. Throughout, females are included in the estimations, and we present separate results by gender. We find sizeable differences in retirement behavior across marital status, gender, labor market attachment, occupation, income, and, in particular, health. We find that the disability retirement exit route that requires specific medical criteria to be met is different from the early retirement route. The latter shares similarities with private pension schemes in a number of countries, including the U.S., where benefits are tied to previous wages, and employers also contribute to this retirement scheme. The differences are pronounced within labor market attachment, income, and in particular health. Furthermore, unemployment followed by early retirement is different from unemployment followed by other programs regarding marital status, gender, income, and health, and is in fact more similar to voluntary early retirement itself. Thus, we have found a portion of unemployment that appears planned. The latter part of the analysis hinges on the competing risk framework. Finally, even when using objective medical diagnosis measures we still find significant effects from health on retirement. Thus, not all health impact on retirement reported in earlier literature was due to justification bias. 


\section{References}

[1] Aalen, O.O.,(1978). "Non parametric inference for a family of counting processes", Annals of Statistics, Vol. 6, pp. 701-726.

[2] An, M.Y., Christensen, B.J. and Datta Gupta, N., (2004). "Multivariate Mixed Proportional Hazard Modelling of the Joint Retirement of Married Couples", Journal of Applied Econometrics, Vol. 19, pp. 687-704.

[3] Anderson, K. H., and Burkhauser R. V., (1985). "The Retirement-Health Nexus: A New Measure of an Old Puzzle", Journal of Human Resources, Vol. 20, no. 3, pp. 315-330.

[4] Antolin, P. and Scarpetta (1998). "Microeconomic Analysis of the Retirement Decision: Germany", Working Paper no. 204, Paris: OECD Economics Department.

[5] Baker, M., Stabile, M. and Deri, C., (2004). "What Do Self-Reported, Objective, Measures of Health Measure?", Journal of Human Resources, Vol. 39, no. 4, pp. 1067-1093.

[6] Benitez-Silva, H., Buchinsky, M., Chan, H-M., Rust, J. , and Sheidvasser, S., (2004). "How Large is the Bias in Self-Reported Disability Status?", Journal of Applied Econometrics, Vol. 19, no. 6, pp. 649-670.

[7] Berndt, E., B. Hall, R. Hall, and J. Hausman, (1974). "Estimation and Inference in Nonlinear Structural Models", Annals of Social Measurement, Vol. 3, pp. 653-665.

[8] Blau, D. M. (1997). "Social Security and the Labor Supply of Older Married Couples", Labor Economics, Vol. 4, pp. 373-418.

[9] Bound, J., (1991). "Self-Reported Versus Objective Measures of Health in Retirement Models", Journal of Human Resources, Vol. 26, no. 1, pp. 106-38.

[10] Breslow, N. (1974). "Covariance Analysis of Censored Survival Data", Biometrics, Vol. 30, No. 1, pp. 89-99.

[11] Broyden, C. G., (1970). "The Convergence of a Class of Double-rank Minimization Algorithms", Journal of the Institute of Mathematics and Its Applications, Vol. 6, pp. 76-90.

[12] Butler, J. S., Anderson, K. H., and Burkhauser, R. V. (1989). "Work and Health after Retirement: A Competing Risk Model with Semiparametric Unobserved Heterogeneity", Review of Economics and Statistics, Vol. 71, no. 1, pp. 46-53.

[13] Cox, D. R. (1972). "Regression models and life-tables", Journal of the Royal Statistical Society, Series B, Vol. 34, pp. 187-220.

[14] Cox, D. R. (1975). "Partial likelihood", Biometrika, Vol. 62, pp. 269-276.

[15] Datta Gupta, N. and Larsen, M., (2010). "The Impact of Health on Individual Retirement Plans: Selfreported vs. Diagnostic Measures", Health Economics, Vol. 19,pp. 792-813. 
[16] Diamond, P. A. and Hausman, J. A. (1984). "The Retirement and Unemployment Behavior of Older Men", In: H. Aaron and G. Burtless (eds.), Retirement and Economic Behavior, Washington, DC: Brookings Institution.

[17] Dwyer, D. and Mitchell, O. S., (1999). "Health Problems as Determinants of Retirement: Are Self-Rated Measures Endogenous?", Journal of Health Economics, Vol. 18, no. 2, pp. 173-93.

[18] Efron, B. (1977). "The efficiency of Cox's likelihood function for censored data", Journal of the American Statistical Association, Vol. 72, No. 359, pp. 557-565.

[19] Fields, G. S. and Mitchell, O. S. (1984). Retirement, pensions and social security. Cambridge: MIT Press.

[20] Fletcher, R., (1970). "A New Approach to Variable Metric Algorithms", Computer Journal, Vol. 13, pp. $317-322$.

[21] Goldfarb, D., (1970). "A Family of Variable Metric Updates Derived by Variational Means", Mathematics of Computation, Vol. 24, pp. 23-26.

[22] Greenwood, M. (1926). "The Natural Duration of Cancer", Reports on Public Health and Medical Subjects, London: Her Majesty's Stationery Office, Vol. 33, pp. 1-26.

[23] Han, A. and Hausman, J. (1990). "Flexible parametric estimation of duration and competing risk models", Journal of Applied Econometrics, Vol. 5, pp. 1-28.

[24] Heckman, J. J. and Singer, B. (1984a). "A method for minimising the impact of distributional assumptions in econometric models for duration data", Econometrica, Vol. 52, pp. 271-320.

[25] Heckman, J. J. and Singer, B. (1984b). "Econometric Duration Analysis", Journal of Econometrics, Vol. 24, pp. 63-132.

[26] Heyma, A., (2004). "A structural dynamic analysis of retirement behaviour in the Netherlands", Journal of Applied Econometrics, Vol. 19, no. 6, pp. 739-759.

[27] Hurd, M. D. (1990). "Research on the Elderly: Economic Status, Retirement and Consumption and Saving", Journal of Economic Literature, Vol. 28, pp. 565-637.

[28] Jenkins, S. P. (2001). Survival analysis with stata (Lecture notes). Essex: Institute of Social and Economic Research.

[29] Johansen, S. (1978). "The product limit estimator as maximum likelihood estimator", Scandinavian Journal of Statistics, Vol. 5, pp. 195-199.

[30] Kalbfleisch, J. D. and Prentice, R. L. (1973). "Marginal likelihoods based on Cox's regression and life model", Biometrika, Vol. 60, pp. 267-278

[31] Kaplan, E.L. and Meier, P. (1958). "Nonparametric Estimation for Incomplete Observations", Journal of the American Statistical Association, Vol. 53, no. 282, pp. 457-481. 
[32] Kiefer, N. (1988). "Economic duration data and hazard functions", Journal of Economic Literature, Vol. 26, pp. 646-679.

[33] Kiefer, N. (1990). "Econometric methods for grouped duration data", In: Panal Data and Labor Market Studies. North-Holland, pp. 97-117.

[34] Lancaster, T. (1979). "Econometric methods for duration of unemployment", Econometrica, Vol. 47, pp. 939-956.

[35] Lancaster, T. (1990). The econometric analysis of transition data. Cambridge University Press.

[36] Lindeboom, M. (1998). "Microeconomic Analysis of the Retirement Decision: The Netherlands", Working Paper no. 207, Paris: OECD Economics Department.

[37] Lindsay, B. G. (1983a). "The geometry of mixture likelihoods: A general theory", Annals of Statistics, Vol. 11, no. 1 , pp. 86-94.

[38] Lindsay, B. G. (1983b). "The geometry of mixture likelihoods, part II: The exponential family", Annals of Statistics, Vol. 11, no. 3, pp. 783-792.

[39] MaCall, B. P. (1994). "Testing the Proportional Hazard Model in the Presence of Unmeasured Heterogeneity", Journal of Applied Econometrics, Vol. 9, pp. 714-733.

[40] McGarry, K., (2004). "Health and Retirement: Do Changes in Health Affect Retirement Expectations?", Journal of Human Resources, Vol. 39, no. 4, pp. 624-64

[41] Meyer, B.D., (1990). "Unemployment insurance and unemployment spells", Econometrica, Vol. 58, no. 4, pp. $757-782$.

[42] Muennig, P., (2005). "Health returns to education interventions", Paper prepared for the Symposium on the Social Costs of Inadequate Education at Columbia University. New York.

[43] Nelson, W., (1972). "Theory and applications of hazard plotting for censored failure data", Technometrics, Vol.14, pp. 945-966.

[44] Nickell, S., (1979). "Estimating the probability of leaving unemployment", Econometrica, Vol. 47, pp. $1249-1266$.

[45] Pedersen, P. J. and Smith, N. (1991). "A Duration Analysis of the Decesion to Retire Early", Working Paper 92-2, Centre for Labor Economics, University of Aarhus and Aarhus School of Business.

[46] Prentice, R. and Gloeckler, L. (1978). "Regression analysis of grouped survival data with application to breast cancer data", Biometrics, Vol. 34, pp. 57-67.

[47] Ridder, G. (1987). "The Sensitivity of Duration Models to Misspecified Unobserved Heterogeneity and Duration Dependence", working paper, University of Groningen. 
[48] Shanno, D. F., (1970). "Conditioning of Quasi-Newton Methods for Function Minimization", Mathematics of Computation, Vol. 24, pp. 647-656.

[49] Sueyoshi, G. T. (1992). "Semiparametric proportional hazards estimation of competing risk models with time-varying covariates", Journal of Econometrics, Vol. 51, pp. 25-58.

[50] Wong, M., Shapiro, M., Boscardin, W. and Ettner, S., (2002). "Contribution of major diseases to disparities in mortality", New England Journal of Medicine,Vol. 347, pp. 1585-1592. 


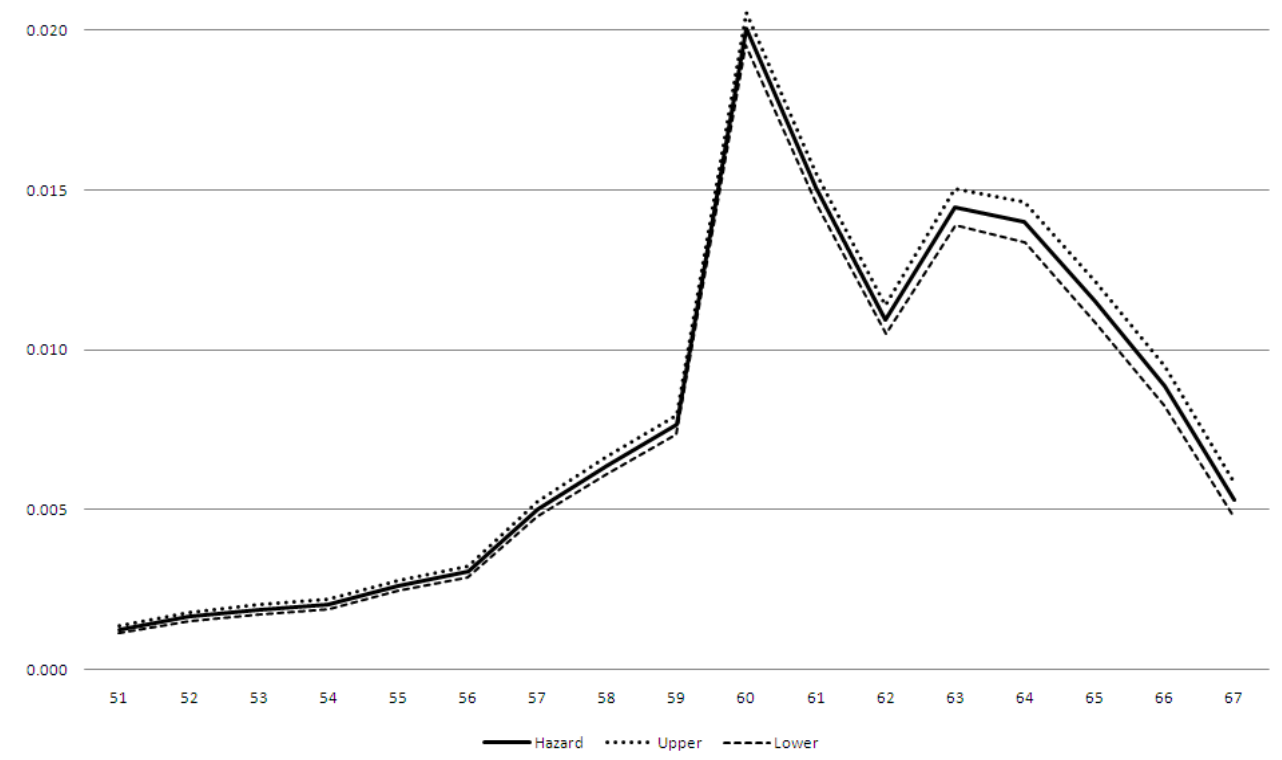

Figure 1: Kaplan Meier estimates for the full population

$$
2
$$

1

0
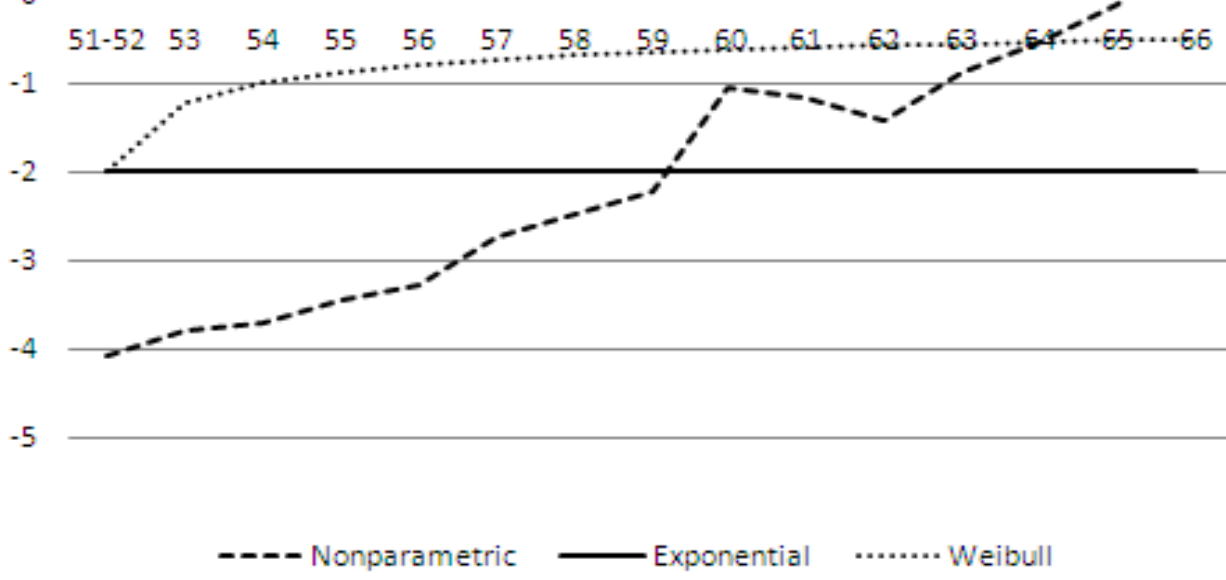

Figure 2: Different specifications of the baseline hazard 


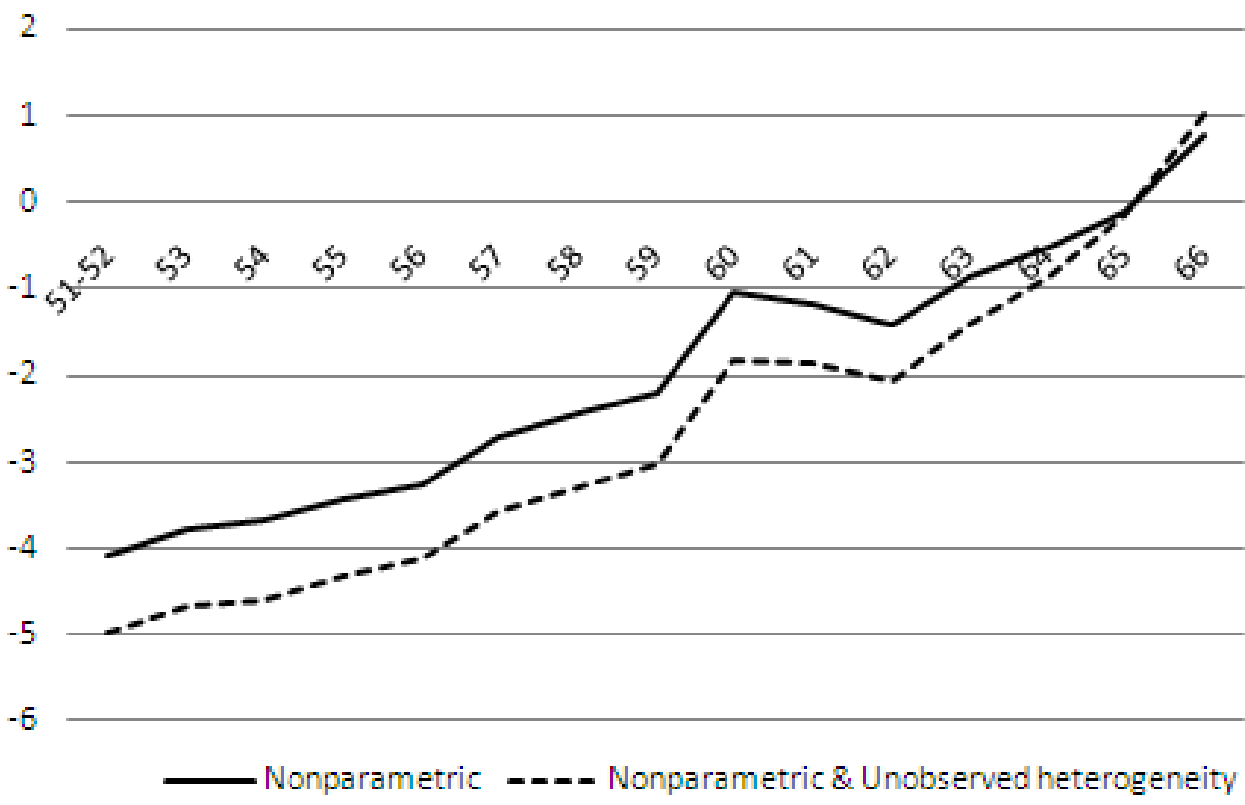

Figure 3: Nonparametric baseline with and without unobserved heterogeneity

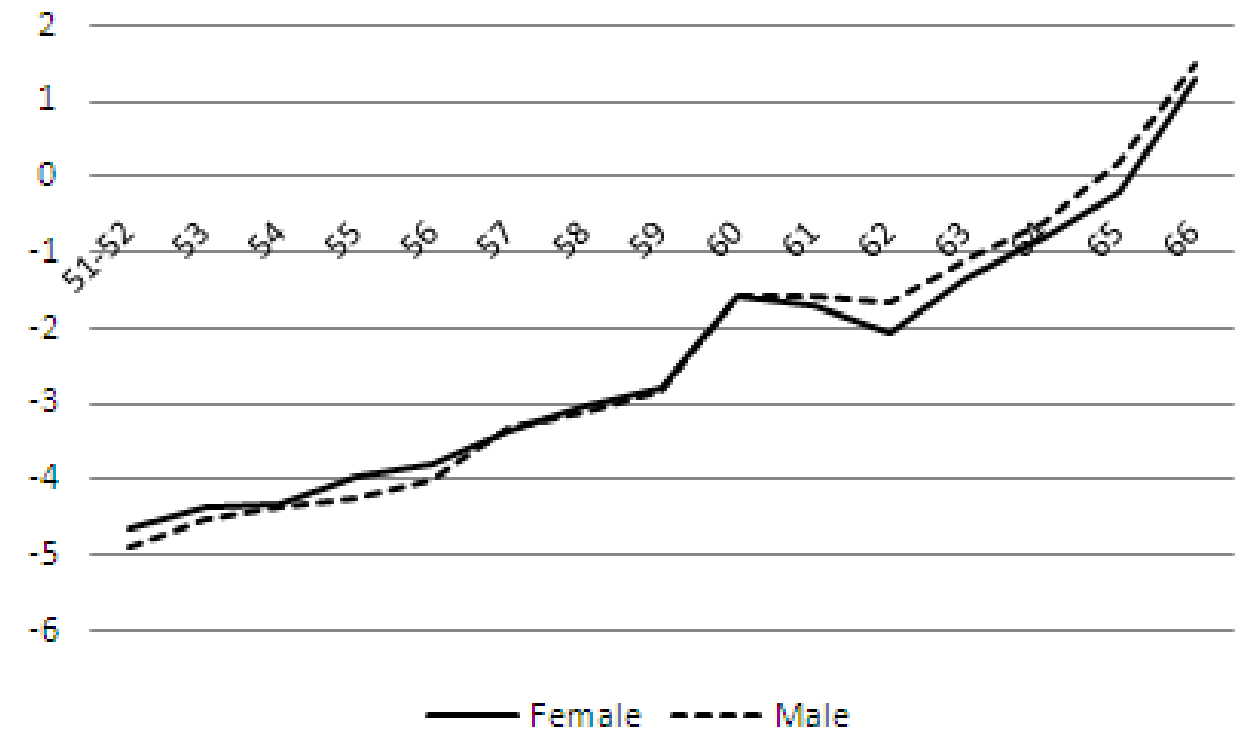

Figure 4: Gender specific nonparametrics baseline with unobserved heterogeneity 


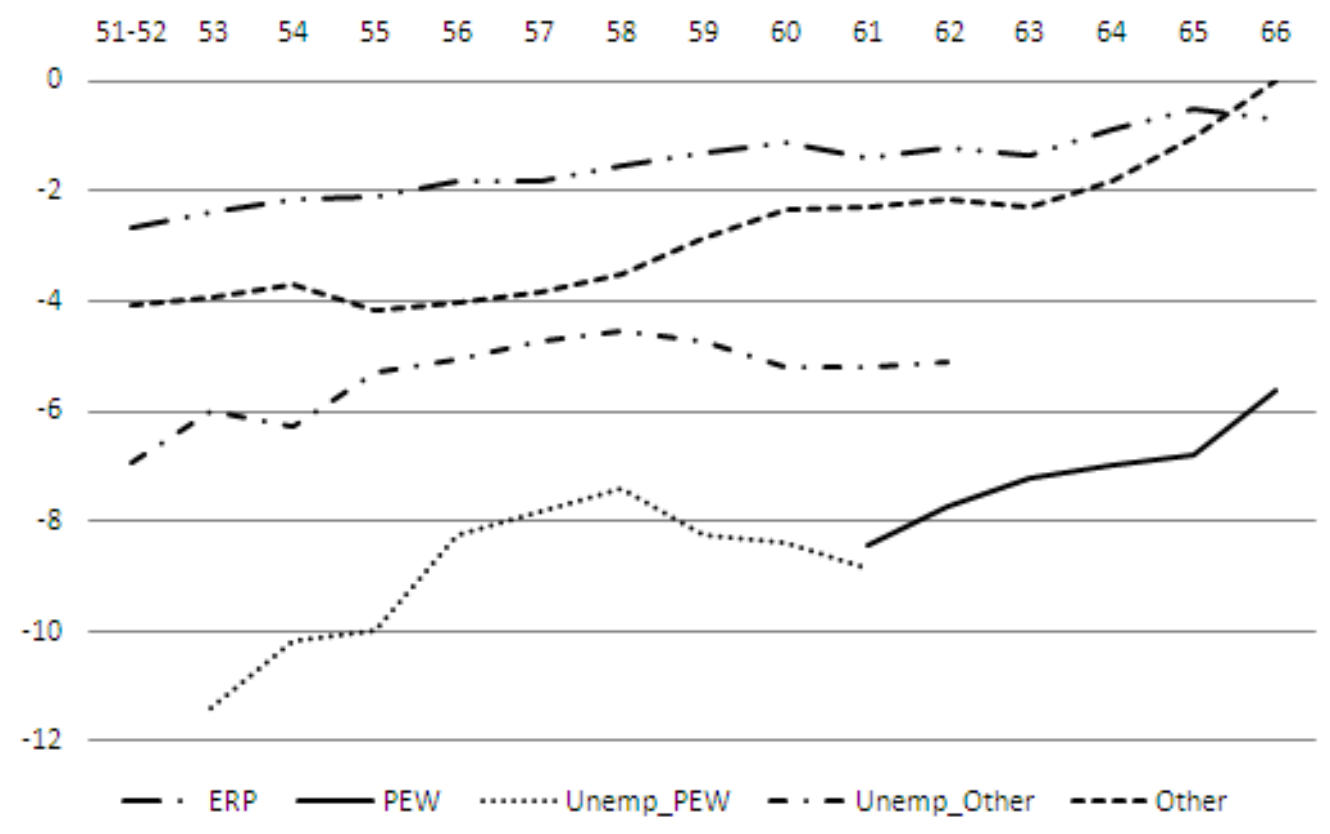

Figure 5: Nonparametric baseline hazards in the competing risk model 
Table 1: Number of spells by duration

\begin{tabular}{lrr}
\hline \hline Duration & Obs. spells & \# of exit \\
\hline 1 - age 51 & 164 & 123 \\
2 - age 52 & 206 & 162 \\
3 - age 53 & 227 & 184 \\
4 - age 54 & 242 & 198 \\
5 - age 55 & 295 & 249 \\
6 - age 56 & 326 & 287 \\
7 - age 57 & 492 & 455 \\
$8-$ age 58 & 603 & 556 \\
9 - age 59 & 695 & 637 \\
$10-$ age 60 & 1,606 & 1,548 \\
$11-$ age 61 & 968 & 925 \\
$12-$ age 62 & 578 & 540 \\
$13-$ age 63 & 675 & 628 \\
$14-$ age 64 & 514 & 479 \\
15 - age 65 & 332 & 314 \\
16 - age 66 & 211 & 187 \\
$17-$ age 67 & 1,195 & 6 \\
\hline Total & 9,329 & 7,478 \\
\hline \hline
\end{tabular}


Table 2: Summary Statistics

\begin{tabular}{lcc}
\hline \hline Panel A & \multicolumn{2}{c}{ All } \\
\hline Variables & Mean & Std.dev. \\
General & & \\
Married & 0.8043 & 0.3967 \\
Male & 0.5660 & 0.4943 \\
City & 0.3508 & 0.4772 \\
Labor Market & & \\
Full time & 0.6640 & 0.4723 \\
Unemployment rate & 0.0573 & 0.1866 \\
Experience & 0.3367 & 0.2370 \\
Member of UI-fund & 0.7738 & 0.4184 \\
Sickness pay & 0.0023 & 0.0475 \\
Education & & \\
Basic & 0.4735 & 0.4985 \\
Vocational & 0.3559 & 0.4788 \\
Short & 0.0262 & 0.1597 \\
Medium & 0.1034 & 0.3045 \\
Long & 0.0399 & 0.1958 \\
Occupation & & \\
Self-employed & 0.1392 & 0.3462 \\
Salaried Worker & & \\
- high level & 0.2343 & 0.4235 \\
- medium level & 0.1762 & 0.3810 \\
- low level & 0.1099 & 0.3128 \\
Unskilled & 0.2458 & 0.4306 \\
Ass. Spouse & 0.0338 & 0.1807 \\
\hline \hline
\end{tabular}


Table 2: (continued) Summary Statistics

\begin{tabular}{lcc}
\hline \hline & \multicolumn{2}{c}{ All } \\
Panel B & Mean & Std.dev. \\
\hline Financial Indicators & 0.6128 & 0.0524 \\
Own income & 0.5763 & 0.0791 \\
Household income & 0.4599 & 0.2612 \\
Wealth & & \\
Health & 0.0072 & 0.0845 \\
Malignant cancer & 0.0066 & 0.0810 \\
Benign cancer & & \\
Endocrine, nutritional & 0.0054 & 0.0734 \\
and metabolic diseases & 0.0018 & 0.0428 \\
Mental and behavioral disorders & & \\
Diseases of the nervous system & & \\
and sensory organs & 0.0048 & 0.0693 \\
Diseases of the circulatory system & 0.0188 & 0.1360 \\
Diseases of the respiratory system & 0.0054 & 0.0735 \\
Diseases of the digestive system & 0.0138 & 0.1168 \\
Diseases of the genitourinary system & 0.0124 & 0.1108 \\
Diseases of the musculoskeletal & & \\
system and connective tissue & 0.0101 & 0.1002 \\
Injury, poisoning, and other & & \\
consequences of external causes & 0.0109 & 0.1037 \\
Other diseases & 0.0149 & 0.1210 \\
\hline \hline
\end{tabular}


Table 3: Maximum likelihood estimates for alternative baseline hazards

\begin{tabular}{|c|c|c|c|c|c|c|}
\hline \multirow{2}{*}{$\begin{array}{l}\text { Panel A } \\
\text { Variables }\end{array}$} & \multicolumn{2}{|c|}{ Exponential } & \multicolumn{2}{|c|}{ Weibull } & \multicolumn{2}{|c|}{ Nonparametric } \\
\hline & Coeff. & Std.err & Coeff. & Std.err & Coeff. & Std.err \\
\hline \multicolumn{7}{|l|}{ General } \\
\hline Married & -0.3169 & 0.0252 & -0.4234 & 0.0244 & -0.0054 & 0.0267 \\
\hline Male & -0.4272 & 0.0263 & -0.3780 & 0.0257 & -0.2674 & 0.0270 \\
\hline City & -0.2522 & 0.0248 & -0.2824 & 0.0246 & -0.0748 & 0.0250 \\
\hline Duration dependence & & & 0.6303 & 0.1962 & & \\
\hline \multicolumn{7}{|l|}{ Labor Market } \\
\hline Full time & -0.4813 & 0.0323 & -0.3484 & 0.0324 & -0.0509 & 0.0367 \\
\hline Unemployment rate & 1.3829 & 0.0521 & 0.9364 & 0.0540 & 1.3679 & 0.0537 \\
\hline Experience & 3.1750 & 0.0594 & 1.6898 & 0.0722 & 0.4556 & 0.0743 \\
\hline Member of UI-fund & 0.3120 & 0.0320 & 0.2909 & 0.0307 & 0.2291 & 0.0380 \\
\hline Sickness pay & 0.8853 & 0.1488 & 0.8638 & 0.1486 & 0.8515 & 0.1214 \\
\hline \multicolumn{7}{|l|}{ Education } \\
\hline Vocational & -0.1417 & 0.0267 & -0.1566 & 0.0263 & -0.0547 & 0.0276 \\
\hline Short & -0.3200 & 0.0783 & -0.3153 & 0.0783 & -0.1898 & 0.0796 \\
\hline Medium & -0.1715 & 0.0474 & -0.2543 & 0.0469 & 0.0589 & 0.0494 \\
\hline Long & -0.3405 & 0.0862 & -0.3979 & 0.0860 & -0.0621 & 0.1110 \\
\hline \multicolumn{7}{|l|}{ Occupation } \\
\hline Self-employed & 0.2517 & 0.0480 & -0.4814 & 0.0530 & 0.7159 & 0.0609 \\
\hline \multicolumn{7}{|l|}{ Salaried Worker } \\
\hline - medium level & 0.3176 & 0.0394 & 0.2173 & 0.0384 & 0.6549 & 0.0431 \\
\hline - low level & 0.7595 & 0.0412 & 0.6080 & 0.0404 & 0.7812 & 0.0467 \\
\hline Unskilled & 0.5701 & 0.0354 & 0.3856 & 0.0345 & 0.9705 & 0.0409 \\
\hline Assisting spouse & 0.7762 & 0.0806 & 0.1947 & 0.0816 & 0.7503 & 0.0873 \\
\hline
\end{tabular}

Note: Bold figures indicate significance at 5 percent level, italics indicate significance at 10 percent level. 
Table 3: (continued) Maximum likelihood estimates for alternative baseline hazards

\begin{tabular}{|c|c|c|c|c|c|c|}
\hline \multirow{2}{*}{$\begin{array}{l}\text { Panel B } \\
\text { Variables }\end{array}$} & \multicolumn{2}{|c|}{ Exponential } & \multicolumn{2}{|c|}{ Weibull } & \multicolumn{2}{|c|}{ Nonparametric } \\
\hline & Coeff. & Std.err & Coeff. & Std.err & Coeff. & Std.err \\
\hline \multicolumn{7}{|l|}{ Financial Variables } \\
\hline Own income & -4.7744 & 0.1537 & -4.8877 & 0.1395 & -2.1234 & 0.2338 \\
\hline Household income & -1.1643 & 0.1547 & -1.5519 & 0.1411 & 0.7481 & 0.2184 \\
\hline Wealth & 0.0214 & 0.0466 & 0.1257 & 0.0454 & -0.0055 & 0.0462 \\
\hline \multicolumn{7}{|l|}{ Health } \\
\hline Malignant cancer & -0.2906 & 0.1350 & -0.3645 & 0.1362 & -0.6380 & 0.1081 \\
\hline Benign cancer & 0.0805 & 0.1263 & 0.1660 & 0.1262 & 0.3131 & 0.1237 \\
\hline \multicolumn{7}{|l|}{ Endocrine, nutritional } \\
\hline and metabolic diseases & 0.3183 & 0.1192 & 0.3106 & 0.1203 & 0.2170 & 0.1114 \\
\hline Mental and behavioral disorders & 0.3875 & 0.1924 & 0.4562 & 0.1898 & 0.7159 & 0.1450 \\
\hline \multicolumn{7}{|l|}{ Diseases of the nervous system } \\
\hline and sensory organs & 0.3209 & 0.1277 & 0.3421 & 0.1274 & 0.3070 & 0.1110 \\
\hline Diseases of the circulatory system & 0.4207 & 0.0707 & 0.4050 & 0.0707 & 0.3523 & 0.0648 \\
\hline Diseases of the respiratory system & 0.2976 & 0.1160 & 0.2333 & 0.1155 & 0.2899 & 0.0963 \\
\hline Diseases of the digestive system & -0.0053 & 0.0921 & 0.0074 & 0.0922 & -0.0060 & 0.0803 \\
\hline Diseases of the genitourinary system & -0.0450 & 0.0985 & -0.0279 & 0.0984 & 0.0368 & 0.0960 \\
\hline \multicolumn{7}{|l|}{ Diseases of the musculoskeletal } \\
\hline system and connective tissue & 0.5126 & 0.0857 & 0.5153 & 0.0856 & 0.4967 & 0.0730 \\
\hline \multicolumn{7}{|l|}{ Injury, poisoning, and other } \\
\hline consequences of external causes & 0.0720 & 0.0986 & 0.0252 & 0.0986 & 0.1630 & 0.0913 \\
\hline Other diseases & -0.0114 & 0.0840 & -0.0269 & 0.0848 & 0.0619 & 0.0771 \\
\hline \# of days of treatment & 3.9744 & 0.4379 & 4.4405 & 0.4685 & 5.2234 & 0.3597 \\
\hline \# of diagnoses & -6.2328 & 1.7642 & -5.6149 & 1.7880 & -0.7361 & 1.4870 \\
\hline \# of admissions & 4.1066 & 1.0417 & 3.0191 & 1.0606 & -0.2234 & 1.0029 \\
\hline Mean Log-likelihood & \multicolumn{2}{|c|}{-0.24617} & \multicolumn{2}{|c|}{-0.24118} & \multicolumn{2}{|c|}{-0.23852} \\
\hline \# Observations & \multicolumn{2}{|c|}{98,465} & \multicolumn{2}{|c|}{98,465} & \multicolumn{2}{|c|}{78,150} \\
\hline
\end{tabular}

Note: Bold figures indicate significance at 5 percent level, italics indicate significance at 10 percent level. 
Table 4: Maximum likelihood estimates for alternative baseline hazards with unobserved heterogeneity

\begin{tabular}{|c|c|c|c|c|c|c|}
\hline \multirow{2}{*}{$\begin{array}{l}\text { Panel A } \\
\text { Variables }\end{array}$} & \multicolumn{2}{|c|}{ Exponential } & \multicolumn{2}{|c|}{ Weibull } & \multicolumn{2}{|c|}{ Nonparametric } \\
\hline & Coeff. & Std.err & Coeff. & Std.err & Coeff. & Std.err \\
\hline \multicolumn{7}{|l|}{ General } \\
\hline Married & -0.3266 & 0.0270 & -0.5468 & 0.0370 & -0.0758 & 0.0320 \\
\hline Male & -0.4591 & 0.0288 & -0.5521 & 0.0381 & -0.3328 & 0.0328 \\
\hline City & -0.2583 & 0.0339 & -0.2846 & 0.0353 & -0.0763 & 0.0299 \\
\hline Duration dependence & & & 1.3932 & 0.0431 & & \\
\hline \multicolumn{7}{|l|}{ Labor Market } \\
\hline Full time & -0.5035 & 0.0349 & -0.3630 & 0.0453 & -0.0557 & 0.0431 \\
\hline Unemployment rate & 1.4939 & 0.0587 & 1.2830 & 0.0674 & 1.4452 & 0.0620 \\
\hline Experience & 3.4618 & 0.0900 & 2.0770 & 0.1037 & 0.3387 & 0.0879 \\
\hline Member of UI-fund & 0.3056 & 0.0339 & 0.1890 & 0.0454 & 0.2182 & 0.0427 \\
\hline Sickness pay & 0.8945 & 0.1532 & 0.8882 & 0.1784 & 0.7443 & 0.1521 \\
\hline \multicolumn{7}{|l|}{ Education } \\
\hline Vocational & -0.1504 & 0.0282 & -0.2109 & 0.0381 & -0.0570 & 0.0331 \\
\hline Short & -0.3427 & 0.0823 & -0.4762 & 0.1089 & -0.2688 & 0.0932 \\
\hline Medium & -0.1775 & 0.0498 & -0.2958 & 0.0652 & 0.0466 & 0.0585 \\
\hline Long & -0.3638 & 0.0894 & -0.5662 & 0.1107 & -0.2066 & 0.1127 \\
\hline \multicolumn{7}{|l|}{ Occupation } \\
\hline Self-employed & 0.4037 & 0.0601 & 0.0547 & 0.0751 & 0.7195 & 0.0685 \\
\hline \multicolumn{7}{|l|}{ Salaried Worker } \\
\hline - medium level & 0.3560 & 0.0415 & 0.4737 & 0.0482 & 0.7147 & 0.0458 \\
\hline -low level & 0.8258 & 0.0452 & 1.0301 & 0.0538 & 0.9070 & 0.0521 \\
\hline Unskilled & 0.6317 & 0.0391 & 0.7754 & 0.0468 & 1.0749 & 0.0447 \\
\hline Ass. Spouse & 0.8800 & 0.0860 & 0.4753 & 0.1053 & 0.7320 & 0.0986 \\
\hline
\end{tabular}

Note: Bold figures indicate significance at 5 percent level, italics indicate significance at 10 percent level. 
Table 4: (continued) Maximum likelihood estimates for alternative baseline hazards with unobserved heterogeneity

\begin{tabular}{|c|c|c|c|c|c|c|}
\hline \multirow{2}{*}{$\begin{array}{l}\text { Panel B } \\
\text { Variables }\end{array}$} & \multicolumn{2}{|c|}{ Exponential } & \multicolumn{2}{|c|}{ Weibull } & \multicolumn{2}{|c|}{ Nonparametric } \\
\hline & Coeff. & Std.err & Coeff. & Std.err & Coeff. & Std.err \\
\hline \multicolumn{7}{|l|}{ Financial Indicators } \\
\hline Own income & -5.1153 & 0.1832 & -7.8112 & 0.2531 & -2.7722 & 0.2845 \\
\hline Household income & -0.9750 & 0.1751 & -0.8136 & 0.2219 & 0.8773 & 0.2421 \\
\hline Wealth & 0.0329 & 0.0486 & -0.1327 & 0.0595 & -0.0676 & 0.0528 \\
\hline \multicolumn{7}{|l|}{ Health } \\
\hline Malignant cancer & -0.2716 & 0.1362 & -0.2935 & 0.1455 & -0.6596 & 0.1164 \\
\hline Benign cancer & 0.0702 & 0.1281 & 0.1392 & 0.1383 & 0.3119 & 0.1352 \\
\hline \multicolumn{7}{|l|}{ Endocrine, nutritional } \\
\hline and metabolic diseases & 0.3183 & 0.1213 & 0.3469 & 0.1337 & 0.2631 & 0.1162 \\
\hline Mental and behavioral disorders & 0.4021 & 0.1963 & 0.6560 & 0.2143 & 0.7465 & 0.1579 \\
\hline \multicolumn{7}{|l|}{ Diseases of the nervous system } \\
\hline and sensory organs & 0.3204 & 0.1298 & 0.3689 & 0.1420 & 0.3606 & 0.1176 \\
\hline Diseases of the circulatory system & 0.4317 & 0.0720 & 0.4359 & 0.0796 & 0.4141 & 0.0689 \\
\hline Diseases of the respiratory system & 0.3136 & 0.1192 & 0.3290 & 0.1302 & 0.3039 & 0.1045 \\
\hline Diseases of the digestive system & -0.0121 & 0.0935 & -0.0337 & 0.1009 & -0.0087 & 0.0877 \\
\hline Diseases of the genitourinary system & -0.0537 & 0.1001 & -0.0285 & 0.1083 & 0.0073 & 0.0987 \\
\hline \multicolumn{7}{|l|}{ Diseases of the musculoskeletal } \\
\hline system and connective tissue & 0.5042 & 0.0873 & 0.4781 & 0.0974 & 0.5319 & 0.0805 \\
\hline \multicolumn{7}{|l|}{ Injury, poisoning, and other } \\
\hline consequences of external causes & 0.0668 & 0.0999 & 0.0108 & 0.1091 & 0.1629 & 0.0981 \\
\hline Other diseases & -0.0128 & 0.0850 & -0.0266 & 0.0917 & 0.1184 & 0.2877 \\
\hline \# of days of treatment & 4.1471 & 0.4629 & 6.5735 & 0.6630 & 5.4836 & 0.4013 \\
\hline \# of diagnoses & -6.1906 & 1.7710 & -4.5248 & 1.9140 & -0.5431 & 1.5073 \\
\hline \# of admissions & 4.1759 & 1.0675 & 2.3884 & 1.2471 & -0.0803 & 1.1013 \\
\hline Gamma mixture distribution & 0.0787 & 0.0187 & 0.7068 & 0.0364 & & \\
\hline$v_{2}$ & & & & & 1.4039 & 0.1150 \\
\hline$p_{2}$ & & & & & 0.1384 & 0.0269 \\
\hline Mean Log-likelihood & \multicolumn{2}{|c|}{-0.24606} & \multicolumn{2}{|c|}{-0.23799} & \multicolumn{2}{|c|}{-0.23718} \\
\hline \# Observations & \multicolumn{2}{|c|}{98,465} & \multicolumn{2}{|c|}{98,465} & \multicolumn{2}{|c|}{78,150} \\
\hline
\end{tabular}

Note: Bold figures indicate significance at 5 percent level, italics indicate significance at 10 percent level. 
Table 5: Gender specific maximum likelihood estimates using a nonparametric baseline

\begin{tabular}{|c|c|c|c|c|}
\hline \multirow{2}{*}{$\begin{array}{l}\text { Panel A } \\
\text { Variables }\end{array}$} & \multicolumn{2}{|c|}{ Men } & \multicolumn{2}{|l|}{ Women } \\
\hline & Coeff. & Std.err & Coeff. & Std.err \\
\hline \multicolumn{5}{|l|}{ General } \\
\hline Married & -0.2872 & 0.0464 & 0.1409 & 0.0456 \\
\hline City & -0.0378 & 0.0407 & -0.1257 & 0.0433 \\
\hline \multicolumn{5}{|l|}{ Labor Market } \\
\hline Full time & -0.1648 & 0.0838 & -0.0516 & 0.0523 \\
\hline Unemployment rate & 1.2037 & 0.0851 & 1.7062 & 0.0932 \\
\hline Experience & 0.0411 & 0.1155 & 0.7507 & 0.1404 \\
\hline Member of UI-fund & 0.2740 & 0.0614 & 0.1429 & 0.0611 \\
\hline Sickness pay & 0.7259 & 0.2302 & 0.8233 & 0.2025 \\
\hline \multicolumn{5}{|l|}{ Education } \\
\hline Vocational & 0.0072 & 0.0452 & -0.1246 & 0.0488 \\
\hline Short & -0.3206 & 0.1213 & -0.1366 & 0.1476 \\
\hline Medium & -0.0274 & 0.0829 & 0.1646 & 0.0803 \\
\hline Long & -0.2822 & 0.1301 & -0.0596 & 0.2069 \\
\hline \multicolumn{5}{|l|}{ Occupation } \\
\hline Self-employed & 0.2680 & 0.1019 & 1.3198 & 0.1086 \\
\hline \multicolumn{5}{|l|}{ Salaried Worker } \\
\hline - medium level & 0.6192 & 0.0670 & 0.8847 & 0.0678 \\
\hline -low level & 0.8016 & 0.0599 & 1.0199 & 0.1052 \\
\hline Unskilled & 0.9080 & 0.0595 & 1.2993 & 0.0707 \\
\hline Ass. Spouse & 0.0724 & 0.4683 & 1.0420 & 0.1180 \\
\hline
\end{tabular}

Note: Bold figures indicate significance at 5 percent level, italics indicate significance at 10 percent level. 
Table 5: (continued) Gender specific maximum likelihood estimates including nonparametric baseline and unobserved heterogeneity

\begin{tabular}{|c|c|c|c|c|}
\hline \multirow{2}{*}{$\begin{array}{l}\text { Panel B } \\
\text { Variables }\end{array}$} & \multicolumn{2}{|c|}{ Men } & \multicolumn{2}{|c|}{ Women } \\
\hline & Coeff. & Std.err & Coeff. & Std.err \\
\hline \multicolumn{5}{|l|}{ Financial Indicators } \\
\hline Own income & -2.7968 & 0.4196 & -3.1606 & 0.4358 \\
\hline Household income & 0.2349 & 0.2862 & 2.4837 & 0.4481 \\
\hline Wealth & -0.0133 & 0.0744 & -0.0076 & 0.0774 \\
\hline \multicolumn{5}{|l|}{ Health } \\
\hline Malignant cancer & -1.2633 & 0.1973 & -0.2818 & 0.1564 \\
\hline Benign cancer & 0.3346 & 0.1985 & 0.2035 & 0.1755 \\
\hline \multicolumn{5}{|l|}{ Endocrine, nutritional } \\
\hline and metabolic diseases & 0.2088 & 0.1527 & 0.2628 & 0.2104 \\
\hline Mental and behavioral disorders & 0.7296 & 0.2317 & 0.5534 & 0.2301 \\
\hline \multicolumn{5}{|l|}{ Diseases of the nervous system } \\
\hline and sensory organs & 0.3601 & 0.1478 & 0.3691 & 0.2201 \\
\hline Diseases of the circulatory system & 0.2936 & 0.0944 & 0.4452 & 0.1174 \\
\hline Diseases of the respiratory system & 0.2773 & 0.1504 & 0.3977 & 0.1614 \\
\hline Diseases of the digestive system & -0.1458 & 0.1237 & 0.0932 & 0.1350 \\
\hline Diseases of the genitourinary system & -0.2537 & 0.1686 & 0.2083 & 0.1311 \\
\hline \multicolumn{5}{|l|}{ Diseases of the musculoskeletal } \\
\hline system and connective tissue & 0.4159 & 0.1155 & 0.6773 & 0.1234 \\
\hline \multicolumn{5}{|l|}{ Injury, poisoning, and other } \\
\hline consequences of external causes & 0.1308 & 0.1314 & 0.0640 & 0.1642 \\
\hline Other diseases & -0.0175 & 0.1067 & 0.0993 & 0.1310 \\
\hline \# of days of treatment & 5.1349 & 0.5406 & 7.7137 & 0.7296 \\
\hline \# of diagnoses & 0.9148 & 2.7171 & -2.1547 & 2.3126 \\
\hline \# of admissions & 1.7440 & 1.7103 & -1.7770 & 1.4471 \\
\hline$v_{2}$ & 1.6344 & 0.2022 & -1.2570 & 0.1912 \\
\hline$p_{2}$ & 0.0878 & 0.0242 & 0.8532 & 0.0589 \\
\hline Mean Log-likelihood & \multicolumn{2}{|c|}{-0.22361} & \multicolumn{2}{|c|}{-0.25622} \\
\hline \# Observations & \multicolumn{2}{|c|}{42,010} & \multicolumn{2}{|c|}{36,140} \\
\hline
\end{tabular}

Note: Bold figures indicate significance at 5 percent level, italics indicate significance at 10 percent level. 
Table 6: Number of spells by duration and destination

\begin{tabular}{|c|c|c|c|c|c|}
\hline \multirow[t]{2}{*}{ Duration } & \multicolumn{5}{|c|}{ Destination } \\
\hline & Disability & PEW & Unemp.-PEW & Unemp.-Others & Others \\
\hline $1-$ age 51 & 56 & 0 & 2 & 25 & 40 \\
\hline $2-$ age 52 & 94 & 0 & 14 & 21 & 33 \\
\hline $3-$ age 53 & 76 & 0 & 16 & 55 & 37 \\
\hline $4-$ age 54 & 93 & 0 & 30 & 32 & 42 \\
\hline $5-$ age 55 & 81 & 0 & 49 & 88 & 31 \\
\hline $6-$ age 56 & 107 & 0 & 49 & 101 & 30 \\
\hline $7-$ age 57 & 95 & 0 & 229 & 91 & 40 \\
\hline $8-$ age 58 & 108 & 0 & 334 & 67 & 47 \\
\hline $9-$ age 59 & 115 & 0 & 411 & 30 & 80 \\
\hline $10-$ age 60 & 96 & 1214 & 123 & 10 & 105 \\
\hline $11-$ age 61 & 49 & 716 & 63 & 13 & 84 \\
\hline $12-$ age 62 & 58 & 361 & 31 & 12 & 78 \\
\hline $13-$ age 63 & 31 & 520 & 16 & 10 & 51 \\
\hline $14-$ age 64 & 30 & 392 & 0 & 3 & 54 \\
\hline $15-$ age 65 & 23 & 214 & 2 & 0 & 75 \\
\hline $16-$ age 66 & 25 & 105 & 0 & 0 & 57 \\
\hline $17-$ age 67 & 0 & 0 & 1 & 5 & 0 \\
\hline Total & 1137 & 3524 & 1370 & 563 & 884 \\
\hline
\end{tabular}




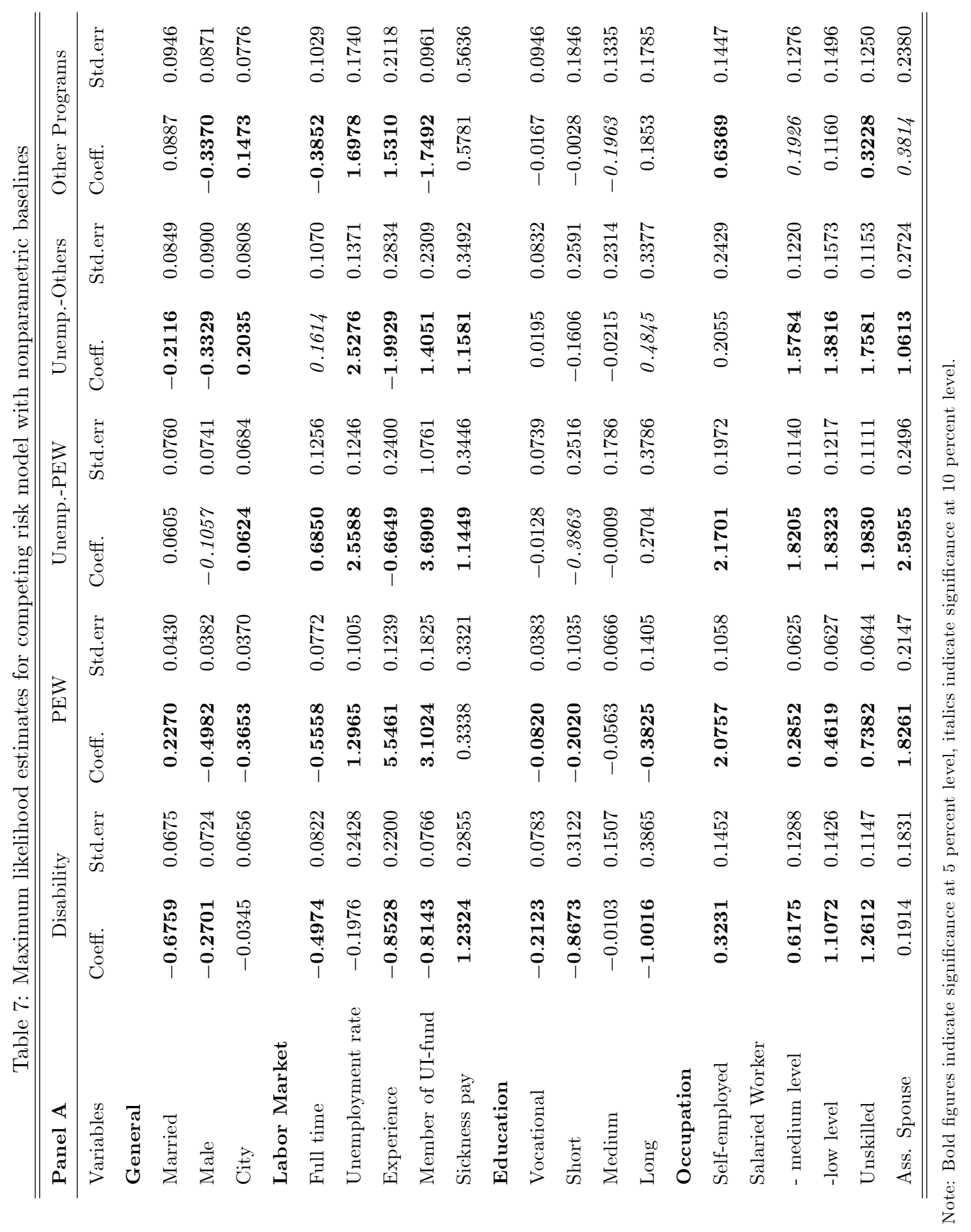




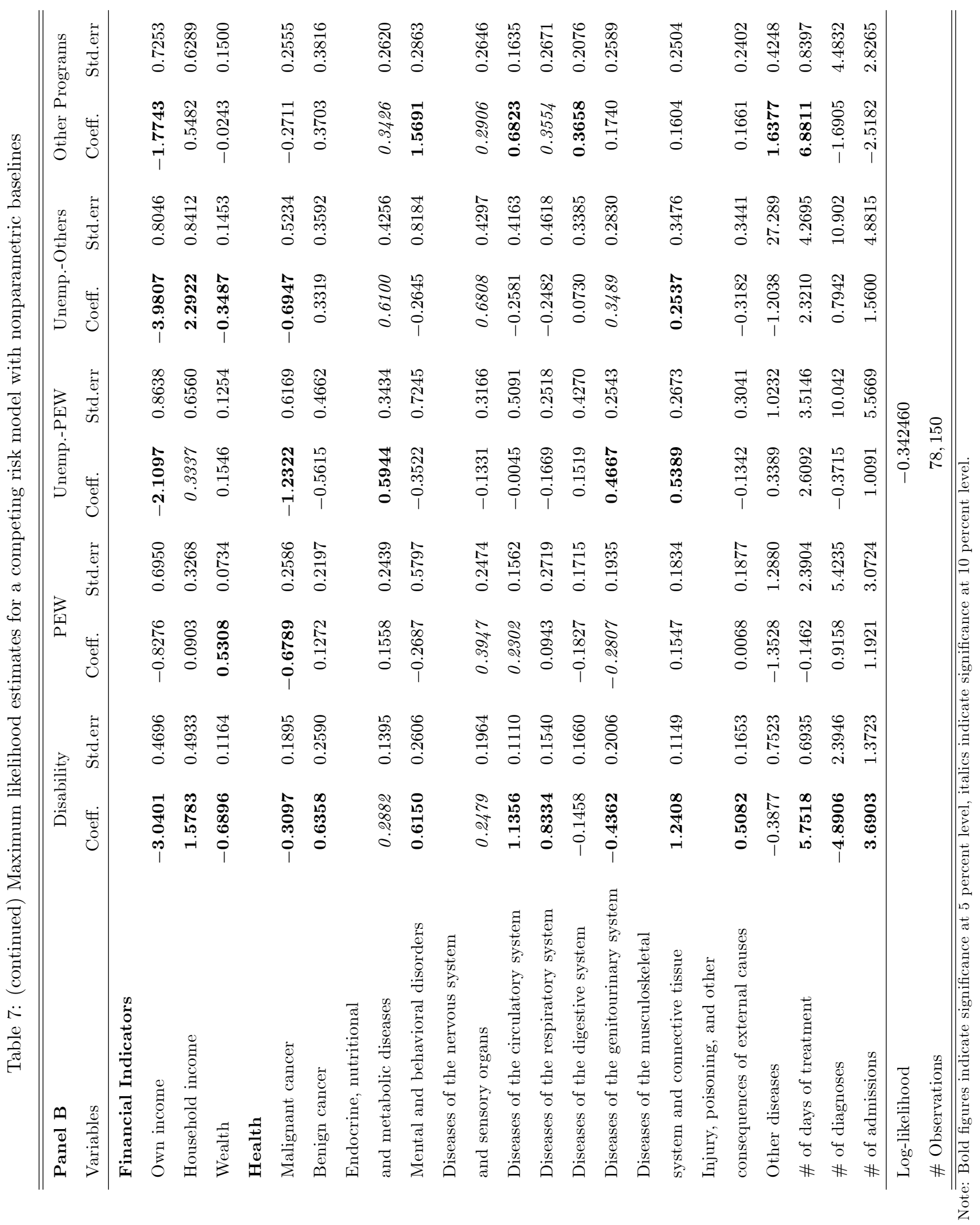




\section{Appendix}

\subsection{Early Retirement Options in Denmark}

Here we briefly describe the relevant retirement options available to the older workers in Denmark.

\subsubsection{Disability}

This is available to people between the ages of 18 and 67 who is permanently unable to work and does not receive any other type of pension. It requires that all attempts to improve the applicant's labor market qualifications, including rehabilitation, treatment, active social policy, etc., have been made. The benefit amount depends on the type of disability pay that is granted. There are four different types. The first one is ordinary disability pay granted to persons between the ages of 60 to 66 years old. It can both be health, social and financial contingent. The second one is increased ordinary disability pay awarded to people between the ages of 18 to 59 again conditioned on either health, social or financial aspects. The benefits are the same as in ordinary disability pay plus an extra supplement. The third is middle disability pay awarded to persons between 18 and 59 years who have occupational skills reduced with more than $2 / 3$. The age group 60-66 years old can also be entitled, but only if an insignificant part of the occupational skill is intact. The amount is the same as ordinary disability pay plus a disablement amount. The final one is highest disability pay awarded to people between 18 and 59 years, whose occupation skills are insignificant. The amount is the same as middle disability pay plus an occupation incompetence amount.

In January 2003 a new law concerning disability came into force. It includes only one type of disability granted to persons between the age of 18 and 65 years. It is conditioned on a significant decrease in the occupational skill such that the individual is unable to become self-supporting in the future - despite the possibilities for public support. It is the old program that is relevant for our sample.

\subsubsection{The Early Retirement (Post Employment Wage) Program}

The post employment wage program (here, simply early retirement) was introduced in 1979 and was initially a political labor market instrument. It was aimed at redistributing work from the older part of the population to the younger part by offering workers between the ages of 60 and 66 the possibility to retire from the labor market and still maintain a reasonable income. The program was justified by two arguments when it was introduced. First it gave workers the possibility to retire early, if they were worn out after many years of psychically and psychologically demanding work. Second, it helped to solve the problem with high rates of youth unemployment in the 1980s.

Early retirement is not awarded on the basis of health conditions like disability, but depends on the degree of labor market participation and type of membership of an approved Unemployment Insurance Fund. There exist two different programs. The first is the New Flexible Post Employment Wage for members that have reached the age of 60 after the 1 July 1999. A person can be eligible in the time frame 60-65 years. The second is the Old Post Employment Wage for members that have reached the age of 60 before the 1 July 1999 and covers the 
age group 60-67 years. Beside the criteria concerning age, the conditions for entitlement and the benefits are different in the two programs. First, if members in the old program did not make use of the early retirement right away and later on get sick, they would loose the right to early retirement. This rule is dropped in the new program. This is a central feature, since workers encompassed by the old program have an incentive to base the retirement decision on uncertainty about the future health situation.

Second, besides paying a contribution to the mandatory membership of an UI-fund, you also have to pay a post employment wage contribution in order to be eligible for the New early retirement. Third, the size of the benefits depends on how the member is insured in the UI-fund. In the old program fully insured will receive 100 percent of the Unemployment Insurance benefit rate for the first two and a half years, but afterward it is reduced to an $82 \%$-rate for the rest of the period. Partly insured will only receive $2 / 3$ of the maximal UI benefit rate and experience same reduction after two and a half year. By postponing the early retirement until age 63 the member will receive the maximum rate the whole period. A person can work 200 hours per year without a reduction in early retirement pay. Current payments from labor market pension schemes will induce a reduction of early retirement pay by 60 percent, if they are paid out. If a person postpones the payments of a pension no reduction will take place. This rule covers all pension schemes with current payments, including rate pension and civil service pension. For capital pension and other total payments, no reduction is made independent of whether or not they are paid out.

In the new program a member will receive 91 percent of the maximal UI benefits if he or she chooses to enter the post employment program within the two first years after the early retirement certificate is issued. This rate will remain unchanged during the whole period. All types of pension schemes will lead to a reduction in early retirement pay independent of whether or not payments are received during the period of early retirement. The rate is still 60 percent. A member will receive the maximum amount of UI benefits (100 percent) during the whole period if he or she waits two years after the certificate is issued to enter the program. This is conditioned on income related work for at least 3,120 hours during that period. Labor market pension with current payments will reduce early retirement pay but only if they are paid out. That is, the old rules apply here. The benefits are the same as the 100 percent rates mentioned in the old program.

Fourth, the new reform also opens up the possibility to earn a tax-free premium. A member who starts the early retirement program at least two years after the early retirement certificate has been issued and who in this period has worked at least 3,120 hours is given the possibility to earn a tax-free premium by working additional 481 hours or more. The maximum a member can earn is 12 x 481 hours, which corresponds to a working period of three years with 37 hours per week. This yields a tax-free premium of DDK 116,628 and should give an incentive to postpone retirement for a couple of years. This premium will be paid when the member reach the age of 65 or dies. Again, it is primarily the old early retirement program that is relevant for our sample. 


\subsubsection{Civil Service Pension (CSP)}

This is a statutory labor market pension scheme for civil servants in government service, which is based on the Public Servants Act. ${ }^{8}$ The size of the pension is based on the salary at the retirement date and the length of the civil servant's employment period. The right to civil service pension starts after ten years of employment as a civil servant and conditioned on that the discharge is caused by age or health. In total a maximum of 37 years can be saved, which will entitle the pensioner to the highest amount of benefits. CSP is part of Other programs in our case.

\subsubsection{Transition Pay (TP)}

Transition pay was a temporary scheme in place between 1992 and 1996. It was offered to unemployed aged 50-60 to leave the labor force for good and in return get $82 \%$ of the highest UI benefits. TP is part of Other programs in our case.

\subsubsection{Unemployment insurance (UI) and Social Assistance (SA)}

Since the unemployment rate in Denmark has been high in some periods, some workers - especially older workers - have had difficulties returning to the labor market after a long unemployment spell. It is therefore reasonable to assume that unemployment could be used as possible retirement route. The benefit a person receives during unemployment depends on how long that person has been unemployed and the previous attachment to the labor market.

Two schemes ensure economic aid to unemployed individuals: Unemployment insurance benefits and social assistance benefits (welfare). The distinction between the two systems is related to the organization of the unemployment insurance system. Membership is voluntary and only members (the insured) will receive UI benefits, when they become unemployed. Non-insured workers will receive SA benefits, which is administered by the municipalities. In order to receive UI benefits, the member must comply with the eligibility criteria. In general this means the member must be available for the labor market and participate in active labor market programs.

Finally, if you are unemployed and have received UI benefits in the last four years you are no longer entitled and must once again earn this right by working. ${ }^{9}$ Otherwise, the member will be transferred to SA benefits. The size of SA-benefits is approximately $3 / 4$ of the maximum UI benefits. However, there is one exception for members between the ages of 55-59 years. If such a member is entitled to Post Employment Wage the benefits can continue until the age of 59 years beyond the normal four years. This means that the member can actually receive benefits for a period of nine years without any contact to the labor market.

SA is part of Other programs in our case.

\footnotetext{
${ }^{8}$ It also includes local authority officers, where the rules are stated in pension regulations.

${ }^{9}$ The eligibility criteria have been strengthened, starting in the eighties. The maximum period one can receive benefits was reduced from seven to four years, and it is was made harder to comply with the eligibility criteria for UI-benefits once they have been lost.
} 
2010-48: Christian M. Dahl and Emma M. Iglesias: Asymptotic normality of the QMLE in the level-effect ARCH model

2010-49: $\quad$ Christian D. Dick, Maik Schmeling and Andreas Schrimpf: Macro Expectations, Aggregate Uncertainty, and Expected Term Premia

2010-50: Bent J esper Christensen and Petra Posedel: The Risk-Return Tradeoff and Leverage Effect in a Stochastic Volatility-in-Mean Model

2010-51: $\quad$ Christos Ntantamis: A Duration Hidden Markov Model for the Identification of Regimes in Stock Market Returns

2010-52: Christos Ntantamis: Detecting Structural Breaks using Hidden Markov Models

2010-53: Christos Ntantamis: Detecting Housing Submarkets using Unsupervised Learning of Finite Mixture Models

2010-54: Stefan Holst Bache: Minimax Regression Quantiles

2010-55: Nektarios Aslanidis and Charlotte Christiansen: Sign and Quantiles of the Realized Stock-Bond Correlation

2010-56: $\quad$ Anders Bredahl Kock: Oracle Efficient Variable Selection in Random and Fixed Effects Panel Data Models

2010-57: Charlotte Christiansen, J uanna Schröter J oensen and J esper Rangvid: The Effects of Marriage and Divorce on Financial Investments: Learning to Love or Hate Risk?

2010-58: Charlotte Christiansen, Maik Schmeling and Andreas Schrimpf: A Comprehensive Look at Financial Volatility Prediction by Economic Variables

2010-59: J ames G. MacKinnon and Morten Ørregaard Nielsen: Numerical distribution functions of fractional unit root and cointegration tests

2010-60: Bent J esper Christensen and Paolo Santucci de Magistris: Level Shifts in Volatility and the Implied-Realized Volatility Relation

2010-61: Christian Bach and Bent J esper Christensen: Latent Integrated Stochastic Volatility, Realized Volatility, and Implied Volatility: A State Space Approach

2010-62: Bent J esper Christensen and Malene Kallestrup Lamb: The Impact of Health Changes on Labor Supply: Evidence from Merged Data on Individual Objective Medical Diagnosis Codes and Early Retirement Behavior 